\title{
Communication Over a Wireless Network With Random Connections
}

\author{
Radhika Gowaikar, Student Member, IEEE, Bertrand Hochwald, Senior Member, IEEE, and Babak Hassibi
}

\begin{abstract}
A network of nodes in which pairs communicate over a shared wireless medium is analyzed. We consider the maximum total aggregate traffic flow possible as given by the number of users multiplied by their data rate. The model in this paper differs substantially from the many existing approaches in that the channel connections in this network are entirely random: rather than being governed by geometry and a decay-versus-distance law, the strengths of the connections between nodes are drawn independently from a common distribution. Such a model is appropriate for environments where the first-order effect that governs the signal strength at a receiving node is a random event (such as the existence of an obstacle), rather than the distance from the transmitter. It is shown that the aggregate traffic flow as a function of the number of nodes $n$ is a strong function of the channel distribution. In particular, for certain distributions the aggregate traffic flow is at least $\frac{n}{(\log n)^{d}}$ for some $d>0$, which is significantly larger than the $O(\sqrt{n})$ results obtained for many geometric models. The results provide guidelines for the connectivity that is needed for large aggregate traffic. The relation between the proposed model and existing distance-based models is shown in some cases.
\end{abstract}

Index Terms-Ad-hoc networks, random connections, throughput.

\section{INTRODUCTION}

A N EARLY study of traffic flow in shared-medium wireless networks appears in the seminal work of Gupta and Kumar [11]. They show that in a grid network of $n$ nodes on the plane having a deterministic power scaling law, $O(\sqrt{n})$ transmitters can talk simultaneously to randomly chosen receivers. Similar results for networks with randomly placed nodes can also be obtained (see, for example, [10] for a recent account). Different models can yield somewhat different conclusions [1], [3], [5], [9], [12], [14]-[17]; nevertheless, if we do not permit the transmitter/receiver pairs to approach one another [6], or for very low attenuation laws [15], the model of a power decay law (as a function of distance) seems to yield a network in which the number of nodes that can talk simultaneously grows much slower than $n$. Network models that incorporate channel fading

Manuscript received June 23, 2005; revised January 30, 2006. This work was supported in part by the National Science Foundation under Grants CCR0133818 and CCR-0326554, by the David and Lucille Packard Foundation, and by Caltech's Lee Center for Advanced Networking. This work was completed while B. Hochwald was with Bell Laboratories, Lucent Technologies.

R. Gowaikar and B. Hassibi are with the Department of Electrical Engineering, California Institute of Technology, Pasadena, CA 91125 USA (e-mail: gowaikar@caltech.edu; hassibi@caltech.edu).

B. Hochwald is with Beceem Communications, Santa Clara, CA 94063 USA (e-mail: hochwald@beceem.com).

Communicated by E. Modiano, Associate Editor for Communication Networks.

Digital Object Identifier 10.1109/TIT.2006.876254 as well as geometric path loss have also been proposed [23], [22] but the scaling behavior of these is not much different from that of [11]. We wish to study networks with a different connectivity model.

The $O(\sqrt{n})$ result in [11] has the following heuristic explanation. If a node wishes to transmit directly to a randomly chosen node (whose distance is approximately $O(\sqrt{n})$ away on average), it has two choices: talk directly, or talk through a series of hops. If it tries to talk directly, the transmitter generates energy in a circle of radius $O(\sqrt{n})$ around itself. However, this energy, which is seen by the intended receiver becomes interference for the $O(n)$ other nodes in the circle. Thus, some fraction of the entire network of $n$ nodes is bathed in interference; an undesirable consequence. If it decides instead to talk through hops, the transmitting node can pass its message to a neighbor, who in turn passes it to a neighbor and so on for $O(\sqrt{n})$ hops to the intended receiver. This strategy limits interference to immediate neighbors but ties up $O(\sqrt{n})$ nodes in the hopping process. Although this turns out to be the best strategy, only $O(\sqrt{n})$ simultaneous messages can be passed before all $n$ nodes in the network are involved.

We change the model of the wireless medium from a model based on distance to one based on randomness. In multiantenna links, a linear increase in capacity (in the minimum of the number of transmit/receive antennas) is obtained when the channel coefficients between the transmit and receive antennas are independent Rayleigh-distributed random variables [4], [13]. It is, therefore, now generally believed that a rich scattering environment, once thought to be detrimental to point-to-point wireless communications, may actually be beneficial. We show that a similar effect may hold for the expected aggregate data traffic in a wireless network; certain forms of randomness can be helpful.

There are several reasons why one may choose a random model over one that is based on distance. While distance effects on signal strength are important for nodes that are very near or far from each other, many networks are designed with minimum and maximum distances in mind. Decay laws of the form $1 / r^{m}$ for a fixed $m>0$ may not be relevant for networks of small physical size. Additionally, through the use of automatic gain control, a radio often artificially mitigates distance effects unless the node is saturated (too close) or "dropped out" (too far). Many first-order signal-strength effects in such networks are then due to random fluctuations in the medium, such as Rayleigh and shadow fading. A distance-power model cannot readily account for shadow fading since signal strength at the receiver is determined more by the presence of an obstacle blocking the path to the transmitter than by distance. In addition, recent investigations show that the connectivity of ad hoc networks with channel 
randomness, such as that caused by shadow fading, is similar to the connectivity in a random graph [24]. Some models that consider channel randomness are studied in [25], [26], where it is shown that the resulting random network has some realistic connectivity properties lacking in a purely deterministic model. We are concerned not just with connectivity but also throughput.

We adopt the premise that randomness can have a first-order effect on the behavior of a network. We assume that the channels between nodes are drawn independently from an identical distribution. We allow the distribution of the channel between nodes to be arbitrary and allow it to vary with the number of nodes $n$. Our model covers environments where the the signal strength at a receiving node is governed primarily by a random event (such as the existence of an obstacle). We believe that the study of such wireless networks with random connections is important for three reasons: First, many real wireless networks have a substantial and dominant random component; second, we show that such networks may have qualitatively different traffic scaling laws than the scaling obtained in geometric models; finally, our results give insight into the connectivity that a network should have to allow large aggregate traffic flows. A short version of this paper appears in [27].

In general, any realistic model of a large network should have a model of connectivity that has a balance of randomness and distance-based effects. In [28], one such model is proposed and its throughput is analyzed. Also, [8] uses a "radio model" to show that in the presence of obstructions and irregularities, channels become approximately uncorrelated with one another, and the probability of good links between nodes that are far apart increases in wireless local area networks (WLAN's). The radio model in [8] essentially uses the same independence assumption that we do, but uses distance to determine the probability of a connection link. We show in Section VIII-A.1 how to apply our traffic-flow conclusions to this radio model to determine a favorable distance between nodes.

\section{A. Approach}

We suppose that the connection strengths between the $n$ nodes of the wireless network are drawn independently and identically from a given arbitrary distribution. In geometric networks such as [11], a node may communicate its message in hops to nearby neighbors so that it ultimately reaches the intended destination. In our random model, although there is no geometric notion of a near neighbor, we can find an equivalent of a near neighbor by introducing the notion of "good paths," where connections stronger than a chosen threshold $\beta$ are called good. Transmissions to relays and destinations occur along only good paths. By figuratively drawing a graph whose vertices are all the nodes in the network, yet whose edges are only the good paths, we obtain a specific random graph model called $\mathcal{G}(n, p)$, where an edge between any pair of the $n$ nodes exists with probability $p$. (In our case, $p$ is simply the probability that the connection strength exceeds $\left.\beta_{n}.\right) \mathcal{G}(n, p)$ is a very well-studied object and we leverage some of its known properties to establish disjoint routes between sources and their intended destinations. However since we are analyzing a wireless network, we must also account for the effects of interference between all nodes, including those that do not have good connections between them. Fortunately, our use of the goodness threshold $\beta$ also makes the analysis of message-failures (due to interference and/or noise) tractable. Our analysis yields an achievable aggregate throughput which is a function of the chosen threshold $\beta$. A judicious choice of $\beta$ can maximize this achievable throughput. To complement our achievability results, we also present on some upper bounds on aggregate throughput that show that our results are sometimes tight.

\section{Model of Transmitted AND Received SignAls}

We assume that the wireless network has narrowband flatfading connections whose powers are independent and identically distributed (i.i.d.) according to an arbitrary distribution $f(\cdot)$. Thus, if $h_{i, j}$ is the connection between nodes $i$ and $j$, then the $\gamma_{i, j}=\left|h_{i, j}\right|^{2}$ are i.i.d. random variables with marginal distribution $f\left(\gamma_{i, j}\right)$. For maximum generality, we allow $f(\gamma)=f_{n}(\gamma)$ to be a function of the number of nodes $n$. As an example, consider

$$
f(\gamma)=(1-p) \cdot \delta(\gamma)+p \cdot \delta(\gamma-1)
$$

where $\delta(\cdot)$ is the Dirac delta-function. This distribution is a simple model of a shadow-fading environment where, for any pair of nodes, with probability $p$ there exists a good connection between them (fading causes no loss), and with probability $1-p$ there exists an obstruction (fading causes a complete loss). In a general network of $n$ nodes, we may let $p=p_{n}$ be a function of $n$ to represent changes in the geography or network topology as the network increases in size. Although $\gamma=0$ and $\gamma=1$ are the only possibilities in the distribution (1), we may also introduce values of $\gamma$ that depend on $n$. Fig. 1 pictorially displays an example of wireless terminals whose connections may obey the model (1).

The behavior of such a network varies dramatically with $p$. At the extreme of $p=1$, no paths are ever blocked and all nodes are fully connected to each other. While this situation permits any node to readily talk to any other node in a single hop, the overall network throughput is low because talking pairs generate an enormous amount of interference for the remaining nodes. If many nodes try to talk simultaneously, the overall interference is overwhelming. At the other extreme of $p=0$, everyone is in a deep fade; now interference is minimal. However, no nodes can talk at all (we assume a transmission power limit). Thus, we have competing effects as a function of $p$ : Increasing $p$ benefits the network by improving connectivity thus allowing for shorter hops, but hurts the network by increasing interference to other receivers. We are led to ask: What $p$ is optimal? What is the resulting network aggregate traffic? Is this optimal $p$ likely to be something we encounter naturally? If not, can we induce it artificially? We answer some of these questions but, more generally, we look at how an arbitrary $f_{n}(\gamma)$ affects the traffic.

\section{A. Detailed Model}

Let the network have $n$ nodes labeled $1, \ldots, n$. Every pair of nodes $\{i, j\}(i \neq j)$ is connected by a channel that is denoted by the random variable $h_{i, j}=h_{j, i}$; there are $\left(\begin{array}{l}n \\ 2\end{array}\right)$ channel random variables. The channel strengths, $\gamma_{i, j}=\left|h_{i, j}\right|^{2}$ are drawn i.i.d. 


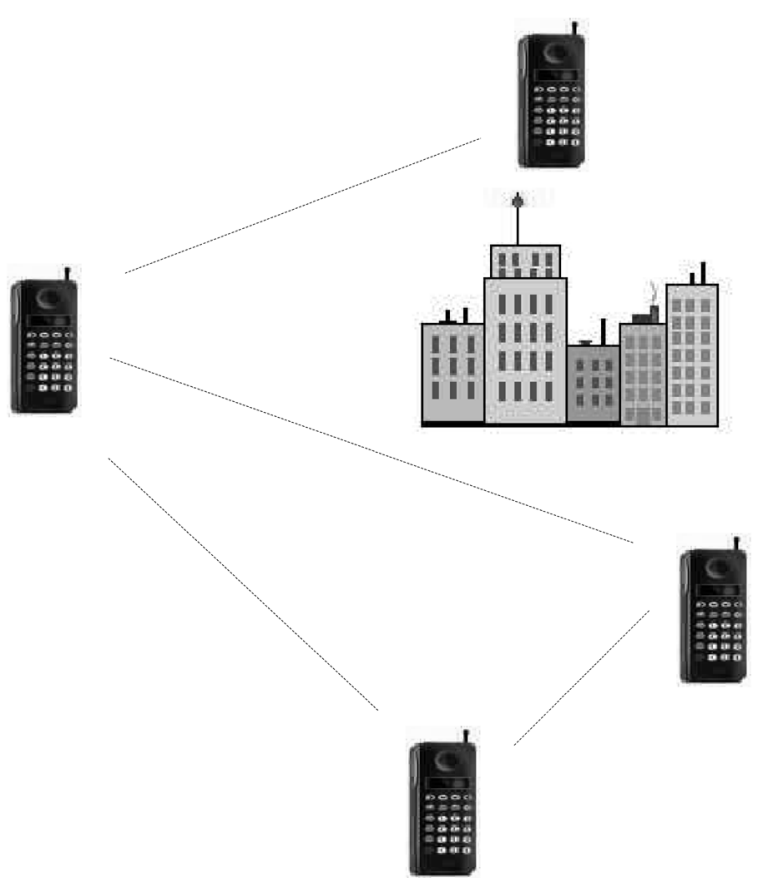

Fig. 1. Nodes are able to establish connections with each other if there is no object in their path. Equation (1) models the presence of an object as a random event where each path has a connection of strength one with probability $p$, and otherwise has a connection of strength zero.

according to the probability density function (pdf) $f_{n}(\gamma)$. Once drawn, these channel variables do not change with time.

Node $i$ wishes to transmit signal $x_{i}$. We assume that $x_{i}$ is a complex Gaussian random process with zero mean and unit variance. Each node is permitted a maximum power of $P$ watts.

We incorporate interference and additive noise in our model as follows. Assume that $k$ nodes $i_{1}, i_{2}, \ldots, i_{k}$ are simultaneously transmitting signals $x_{i_{1}}, x_{i_{2}}, \ldots, x_{i_{k}}$ respectively. Then the signal received by node $j\left(\neq i_{1}, \ldots, i_{k}\right)$ is given by

$$
y_{j}=\sum_{t=1}^{k} \sqrt{P} h_{i_{t}, j} x_{i_{t}}+w_{j}
$$

where $w_{j}$ represents additive noise. The additive noise variables $w_{1}, \ldots, w_{n}$ are i.i.d., drawn from a complex Gaussian distribution of zero mean and variance $\sigma^{2}\left(w_{j} \sim \mathcal{C N}\left(0, \sigma^{2}\right)\right)$. The noise is statistically independent of $x_{i}$.

\section{B. Successful Communication}

In (2), suppose that only node $i_{1}$ wishes to communicate with node $j$ and the signals $x_{i_{2}}, \ldots, x_{i_{k}}$ are interference. Then, the signal-to-interference-plus-noise ratio (SINR) for node $j$ is given by

$$
\rho_{j}=\frac{P \gamma_{i_{1}, j}}{\sigma^{2}+P \sum_{l=2}^{k} \gamma_{i_{l}, j}}
$$

We assume that transmission is successful when the SINR exceeds some threshold $\rho_{0}$. If the SINR is less than $\rho_{0}$ we say that transmission is not possible. Thus, even though $\rho_{j} \geq \rho_{0}$, we

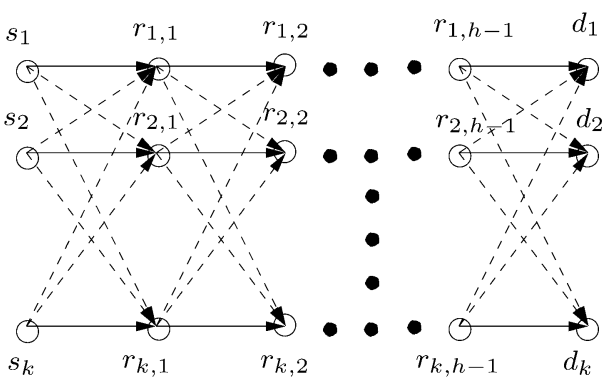

Fig. 2. Schedule of relay nodes: Source $s_{i}$ communicates with destination $d_{i}$ using relays $r_{i, 1}, \ldots, r_{i, h-1}$. The solid lines indicate intended transmissions and the dashed lines indicate potential interference. A schedule is valid if it meets the no-collision conditions that a node can receive or transmit at most one message in any time slot and that no node can transmit and receive simultaneously.

use $\log \left(1+\rho_{0}\right)$ as the transmission rate. Using $\log \left(1+\rho_{0}\right)$ as the rate, rather than the more precise $\log \left(1+\rho_{j}\right)$, simplifies our analysis.

\section{Network OpERATION AND OBJECTIVE}

We suppose that $k$ nodes, denoted by $s_{1}, \ldots s_{k}$, are randomly chosen as sources. For every $s_{i}$, a destination node $d_{i}$ is chosen at random, thus making $k$ source-destination pairs. We assume that these $2 k$ nodes are all distinct and, therefore, $k \leq n / 2$. Source $s_{i}$ wishes to transmit message $M_{i}$ to destination $d_{i}$ and has encoded it as signal $x_{i}$. We wish to see how many source-destination pairs may communicate simultaneously. The sources may talk directly to the destination nodes or may decide to communicate in hops through a series of relay nodes.

\section{A. Communicating With Hops}

In general, we suppose that the source-destination pair $\left(s_{i}, d_{i}\right)$ communicates using a sequence of relay nodes $r_{i, 1}, r_{i, 2}, \ldots, r_{i, h-1}$. ( $h=1,2, \ldots$ represents the number of hops.) Define $r_{i, 0}=s_{i}$ and $r_{i, h}=d_{i}$. The path from $s_{i}$ to $d_{i}$ is then $r_{i, 0}=s_{i}, r_{i, 1}, r_{i, 2}, \ldots, r_{i, h-1}, r_{i, h}=d_{i}$. In time slot $t+1$, we have nodes $r_{1, t}, r_{2, t}, \ldots, r_{k, t}$ transmitting simultaneously to nodes $r_{1, t+1}, r_{2, t+1}, \ldots, r_{k, t+1}$, respectively. We ask that nodes $r_{1, t+1}, r_{2, t+1}, \ldots, r_{k, t+1}$ decode their respective signals $x_{1}, x_{2}, \ldots, x_{k}$ and transmit them to the next set of relay nodes in the $(t+2)$ th time slot, and so on. A natural condition to impose is that the relay nodes that are receiving (or transmitting) messages in any time slot be distinct; the messages do not collide. In addition, we ask that relay nodes not receive and transmit at the same time. We refer to these conditions together as the property of no collisions in the rest of this paper. In general, we do not require $r_{i, t}$ to be distinct from $r_{i, t+1}$ for any $i$. This means that a relay can effectively hold on to a message in a time slot; hence, $h$ effectively represents the maximum number of hops needed for all the source-destination pairs (see Fig. 2).

\section{B. Throughput}

With the aforementionee procedure, we have $k$ simultaneous communications occurring in $h$ time slots. Message $M_{i}$ reaches the intended destination $d_{i}$ successfully if it can be decoded by each relay $r_{i, t}$. Assume that a fraction $1-\epsilon$ of messages 
reach their intended destinations in this way. Then, we define the throughput as

$$
T=(1-\epsilon) \frac{k}{h} \log \left(1+\rho_{0}\right),
$$

where $\rho_{0}$ is the SINR threshold, and we are using the natural logarithm. Thus, $\log \left(1+\rho_{0}\right)$ is the sustainable throughput per user if the users do not collide, as mentioned in Section II-B. We multiply this factor by the number of noncolliding source-destination pairs $k$, divide by the number of hops, and subtract the fraction of dropped messages $\epsilon$. The resulting throughput $T$ depends on $n$ and we sometimes add subscripts to the variables involved to indicate this $k_{n}, \epsilon_{n}, \rho_{0, n}$ and $T_{n}$. Typically, we force $\epsilon_{n}$ to go to zero as $n$ grows. We demonstrate a scheme for choosing the relay nodes and analyze the throughput performance of this scheme. Thus, we give an achievability result for $T_{n}$. We now state this result.

\section{MAIN RESUlT}

Theorem 1: Consider a network on $n$ nodes whose edge strengths are drawn i.i.d. from a pdf $f_{n}(\gamma)$. Let $F_{n}(\gamma)$ denote the cumulative distribution function corresponding to $f_{n}(\gamma)$ and define $Q_{n}(\gamma)=1-F_{n}(\gamma)$. Choose any $\beta_{n}$ such that $Q_{n}\left(\beta_{n}\right)=\frac{\log n+\omega_{n}}{n}$, where $\omega_{n} \rightarrow \infty$ as $n \rightarrow \infty$. Then, there exists a positive constant $\alpha$ such that a throughput of

$$
\begin{aligned}
T=\left(1-\epsilon_{n}\right) \alpha k_{n}\left(\beta_{n}\right) \frac{\log \left(n Q_{n}\left(\beta_{n}\right)\right)}{\log n} & \\
& \times \log \left(1+\frac{a_{n} \beta_{n}}{\frac{\sigma^{2}}{P}+\left(k_{n}\left(\beta_{n}\right)-1\right) \mu_{\gamma}}\right)
\end{aligned}
$$

is achievable for any positive $a_{n}$ such that $a_{n} \leq 1$ and any $k_{n}\left(\beta_{n}\right)$ that satisfy the following conditions:

1)

$$
k_{n}\left(\beta_{n}\right) \leq \alpha n \frac{\log \left(n Q_{n}\left(\beta_{n}\right)\right)}{\log n}
$$

2)

$$
\begin{aligned}
\epsilon_{n} & \leq \frac{a_{n}^{2}}{\alpha\left(1-a_{n}\right)^{2}} \frac{\left(k_{n}\left(\beta_{n}\right)-1\right) \sigma_{\gamma}^{2}}{\left(\frac{\sigma^{2}}{P}+\left(k_{n}\left(\beta_{n}\right)-1\right) \mu_{\gamma}\right)^{2}} \frac{\log n}{\log \left(n Q_{n}\left(\beta_{n}\right)\right)} \\
& \rightarrow 0
\end{aligned}
$$

where $\mu_{\gamma}$ and $\sigma_{\gamma}^{2}$ are the mean and variance of $\gamma$, respectively. The SINR threshold $\rho_{0}$ is given by

$$
\frac{a_{n} \beta_{n}}{\frac{\sigma^{2}}{P}+\left(k_{n}\left(\beta_{n}\right)-1\right) \mu_{\gamma}} .
$$

The parameter $\beta_{n}$ satisfying $Q_{n}\left(\beta_{n}\right)=\frac{\log n+\omega_{n}}{n}$ is the goodness threshold mentioned in Section I-A. By figuratively drawing an edge when $\gamma>\beta_{n}$, we obtain a random graph that fits the well-studied model $\mathcal{G}(n, p)$. Condition (5) is needed to obtain a noncolliding schedule in this random graph. This issue is discussed in detail in Section V. Once the schedule is obtained, we incorporate the effects of interference between noncolliding transmissions and provide an error analysis in Section VI. Condition (6) forces $\epsilon_{n}$ to go to zero. In Section VII, we combine the results of Sections V and VI to prove the theorem. Note that the theorem indicates an achievable throughput and does not preclude that higher throughputs are possible.

Although it is not evident from the theorem statement, it turns out that the optimum number of hops $h$ grows at most logarithmically with $n$. The throughput, therefore, depends most strongly on the number of simultaneous transmissions $k_{n}$ and the SINR threshold $\rho_{0}$.

The throughput expression (4) is general and accommodates an arbitrary $f_{n}(\gamma)$. The parameter $k_{n}$ is the number of noncolliding simultaneous transmissions. We discuss the constant $\alpha$ and the parameter $a_{n}$ later. The joint selection of $\beta_{n}, k_{n}$, and $a_{n}$ that maximizes the achievable throughput (4) is not easily expressed in closed-form as a function of the pdf $f_{n}(\gamma)$. In general, these parameters need to be determined on a case-by-case basis. We show how to find the necessary parameters in Section VIII where we give several examples.

Since (4) holds for any $k_{n}$ satisfying (5), we may choose $k_{n}$ as large as possible [achieving equality in (5)] and optimize only over $a_{n}$ and $\beta_{n}$. In fact, when $\frac{\sigma^{2}}{P}-\mu_{\gamma} \geq 0$, it is possible to show that the optimum $k_{n}$ is the maximum possible. Hence, we state a more specific achievability result.

Corollary 1: In the network of Theorem 1 , if $\frac{\sigma^{2}}{P}-\mu_{\gamma} \geq 0$ the throughput (4) is maximized by choosing $k_{n}$ as large as possible.

At this point, we would like to refer back to the problem setting of [11] and note that their model of a random network, where nodes wish to send information at the rate of $\lambda(n)$ bits per second to a randomly chosen destination is closest to the problem we consider here. For the random network, an aggregate throughput capacity of $O(\sqrt{n / \log n})$ is obtained in [11]. (This is only slightly worse than the transport capacity of $O(\sqrt{n})$ for the somewhat different model of arbitrary networks, which has been discussed in the introduction to this work.) In the example presented in Section VIII-B, we examine the scaling behavior of the throughput with a pdf $f_{n}(\gamma)$ that is obtained based on a distance-decay law. The effects of doing away with the geometric model become more clear with that example.

\section{SCHEDULING TRANSMISSIONS}

With a view to meeting a minimum SINR of $\rho_{0}$ at every relay node at every hop, we impose the condition that each transmitting link be stronger than some threshold $\beta_{n}$. We require that $\gamma_{r_{i, t}, r_{i, t+1}} \geq \beta_{n}$, where $\beta_{n}$ is a design parameter. We denote links that satisfy $\gamma_{i, j} \geq \beta_{n}$ as good. We require the path from $s_{i}$ to $d_{i}$ to use only good links.

The threshold $\beta_{n}$ is a parameter that we may choose as a compromise between quantity and quality of the connections. By making $\beta_{n}$ large, we increase the quality of the link. However, if we make it too large we risk not being able to form an uninterrupted path of good links from the source to the destination. 
In this section, we determine the relation between $\beta_{n}$ and the lengths of source-destination paths.

Define $p_{n}=P\left(\gamma \geq \beta_{n}\right)$ (for convenience, we drop the subscript $n$ in the rest of this section). Using our wireless communication network, we define a graph on $n$ vertices as follows: For (distinct) vertices $i$ and $j$ of the graph, draw an edge $(i, j)$ if and only if $\gamma_{i, j} \geq \beta_{n}$ in the network. Call the resulting graph $G(n, p)$. The graph $G(n, p)$ then becomes an instance of a model called $\mathcal{G}(n, p)$ on $n$ vertices in which edges are chosen independently and with probability $p[2]$. This graph shows the possible paths from the various sources to the various destinations using only good links, but does not show the possible interference encountered if these paths are used simultaneously. We examine this interference in Section VI.

Graphs taken from the model $\mathcal{G}(n, p)$ have many known properties. For instance, the values of $p$ for which the graph is connected is well characterized. As $p$ increases, the probability that the graph is connected goes to one. If $p=\frac{\log n+c+o(1)}{n}$ (where $c>0$ need not be a constant) the probability of the graph being connected is $e^{-e^{-c}}$ [2]. This implies that there is a phase transition in the graph around $p=\frac{\log n}{n}$. For $p$ less than this, the probability of connectivity goes to zero rapidly and for $p$ greater than this it goes to one rapidly. Another property that is well studied is the diameter. The diameter of a graph is defined as the maximum distance between any two vertices of the graph, where the distance between two vertices is the minimum number of edges one has to traverse to go from one to the other. Results in [2] and [18] tell us that for $p$ in the range of connectivity the diameter behaves like $\frac{\log n}{\log n p}$. (It is also known that the average distance between two nodes has the same behavior.) This tells us that a message can be transmitted from one node to another using at most $\frac{\log n}{\log n p}$ hops. What it leaves unanswered is the question of how to establish $k$ such transmissions simultaneously and on noncolliding paths.

The problem of obtaining a noncolliding schedule can be thought of more generally as a problem of avoiding or reducing interference. Not surprisingly, several works that study throughput scaling in large networks encounter the same issue, irrespective of the precise network model being employed. For instance, in [11], the number of routes that pass through a certain small area of the network (which they call a cell) can be thought of as the bottleneck that determines the overall throughput. Similarly, in [10], the number of disjoint paths that can be found in a certain area can be perceived as the limiting factor. Various techniques are used in these works to enable this calculation. While [11] uses results relating to the Vapnik-Chervonenkis dimension, [10] uses ideas inspired by percolation theory and random geometric graphs [29]. In the setting of this work, it is most natural to use random graph theory, and we use a relatively recent result regarding vertex-disjoint paths by Broder et al. [19] in order to find a satisfactory noncolliding schedule.

\section{A. Scheduling Using Vertex-Disjoint Paths in $G(n, p)$}

Two paths that do not share a vertex are called vertex-disjoint. Note that any two paths that are vertex-disjoint satisfy our "no-collisions" property; however, the reverse statement is not true. Thus, the vertex-disjoint condition is stronger than our requirement of noncolliding paths. For a set of $k$ (disjoint) pairs of vertices $\left(s_{i}, d_{i}\right)$, the question of whether there exists a set of vertex-disjoint paths connecting them is addressed in [19]. Their result states that with high probability, for every (sufficiently random) set of $k$ pairs $\left(s_{i}, d_{i}\right)$ and $k$ not greater than $\alpha_{1} n \frac{\log n p}{\log n}$ where $\alpha_{1}$ is a constant, there exists a set of vertex-disjoint paths. This result is within a constant of the best one can hope to achieve since the average distance between nodes in $\mathcal{G}(n, p)$ is $\frac{\log n}{\log n p}$ and, thus, we can certainly have no more than $n \frac{\log n p}{\log n}$ vertex-disjoint paths. Also stated in [19] is an algorithm that finds $k$ paths using various random walk and flow techniques. Here, we reproduce their main result.

Theorem 2: Suppose that $G=G(n, p)$ and $p \geq \frac{\log n+\omega_{n}}{n}$, where $\omega_{n} \rightarrow \infty$. Then, there exist two positive constants $\alpha_{1}$, $\alpha_{2}$ such that, with probability approaching 1 , there are vertexdisjoint paths connecting $s_{i}$ to $d_{i}$ for any set of pairs

$$
F=\left\{\left(s_{i}, d_{i}\right) \mid s_{i}, d_{i} \in\{1, \ldots, n\}, i=1, \ldots, k\right\}
$$

satisfying the following.

1) The pairs in $F$ for $i=1, \ldots, k$ are disjoint.

2) The total number of pairs, $k=|F|$, is not greater than $\alpha_{1} n \frac{\log n p}{\log n}$.

3) For every vertex $v \in\{1, \ldots, n\}$, no more than a $\alpha_{2}$-fraction of its set of neighbors, $N(v)$, are prescribed endpoints, that is $|N(v) \cap(S \cup D)| \leq \alpha_{2}|N(v)|$, where $S=\left\{s_{i}\right\}$ and $D=\left\{d_{i}\right\}$.

Furthermore, these paths can be constructed by an explicit randomized algorithm in polynomial time.

In fact, the existence of the paths is proved by stating and analyzing a randomized algorithm that finds them. However, we use this theorem only as an existence result to demonstrate achievable throughputs. Some comments about their randomized algorithm can be found in Sections VI and X-A.

In our communication network, condition 1$)$ that $\left(s_{i}, d_{i}\right)$ be disjoint pairs is already met. The second imposes a restriction on how large $k$ can be. Since the $k$ source-destination pairs are chosen at random, the third condition is also met. (In fact, the third condition is imposed in [19] to prevent someone from choosing the $\left(s_{i}, d_{i}\right)$ pairs in a particularly adversarial manner using knowledge of the graph structure.)

We can restate the theorem for our purposes.

Theorem 3: Suppose that $G=G(n, p)$ and $p \geq \frac{\log n+\omega_{n}}{n}$, where $\omega_{n} \rightarrow \infty$. Then there exists a constant $\alpha>0$ such that, with probability approaching 1 , there are vertex-disjoint paths connecting $s_{i}$ to $d_{i}$ for any set of disjoint, randomly chosen source-destination pairs

$$
F=\left\{\left(s_{i}, d_{i}\right) \mid s_{i}, d_{i} \in\{1, \ldots, n\}, i=1, \ldots, k\right\}
$$

provided $k=|F|$ is not greater than $\alpha n \frac{\log n p}{\log n}$.

The constant $\alpha$ in this theorem is the same $\alpha$ required in Theorem 1. It is not explicitly specified. We examine the lengths that these $k$ paths can have in the following lemma. 
Lemma 1: Almost all of the $k=\alpha n \frac{\log n p}{\log n}$ vertex-disjoint paths obtainable under Theorem 3 have lengths that grow no faster than $\frac{\log n}{\alpha \log n p}$.

Proof: Suppose that some fraction of paths, say $c_{n} k$ where $c_{n}>0$ have average lengths of the form $\frac{\log n}{\log n p}\left(1+\omega_{n}^{\prime}\right)$ where $\omega_{n}^{\prime}$ goes to infinity. Since there are $n$ nodes in the network, we have

$$
\begin{aligned}
n & \geq c_{n} k \times \frac{\log n}{\log n p}\left(1+\omega_{n}^{\prime}\right) \\
& =c_{n} \alpha n \frac{\log n p}{\log n} \times \frac{\log n}{\log n p}\left(1+\omega_{n}^{\prime}\right)=c_{n} \alpha n\left(1+\omega_{n}^{\prime}\right) .
\end{aligned}
$$

This implies that $1 \geq \alpha c_{n}\left(1+\omega_{n}^{\prime}\right)$ and, therefore, $c_{n}$ must go to zero. Therefore, we conclude that at most a vanishing fraction of the $k$ paths can have lengths that grow faster than $\frac{\log n}{\log n p}$ and, asymptotically, all the paths have lengths that grow no faster than $\frac{\log n}{\alpha \log n p}$.

Hence, the number of hops $h$ is (asymptotically) at most $\frac{\log n}{\alpha \log n p}$. We use this fact in the error analysis in Section VI.

\section{PROBABILITY OF ERROR}

Consider a schedule of $k \leq \alpha n \frac{\log n p}{\log n}$ noncolliding paths. Theorem 3 shows that such a schedule exists. One possible (but often impractical) way to obtain such a schedule is to use an exhaustive search that first lists all the paths between every sourcedestination pair and then randomly chooses a set that satisfies the vertex-disjoint property. Because we thereby choose a path based on vertices rather than edges, we are assured that any edges that might exist between vertices along one path to vertices along another are i.i.d. Bernoulli distributed with parameter $p$. We also conclude that the channel connections between nodes along different paths in the network are i.i.d. with distribution $f_{n}(\gamma)$.

More generally, randomized algorithms that choose noncolliding paths without using edge information between such paths also have the property of generating i.i.d. interference between the paths. An example of such a randomized algorithm that avoids an exhaustive search is [19].

We now consider the probability that a particular message fails to reach its intended destination. Destination $d_{i}$ fails to receive message $M_{i}$ if the SINR falls below $\rho_{0}$ at any of the $h$ relay nodes $r_{i, 1}, \ldots, r_{i, h}=d_{i}$. Denote by $E_{t}$ the event that relay node $r_{i, t}$ does have an SINR greater than $\rho_{0}$. Note that the events $E_{1}, \ldots, E_{h}$ are identical. Therefore, we have

$$
\begin{aligned}
P\left(M_{i} \text { is received successfully }\right) & =P\left(\bigcap_{t=1}^{h} E_{t}\right) \\
& =1-P\left(\bigcup_{t=1}^{h} \sim E_{t}\right) \\
& \geq 1-\sum_{t=1}^{h} P\left(\sim E_{t}\right) \\
& =1-h P\left(\sim E_{1}\right)
\end{aligned}
$$

where the inequality comes from the union bound. We now compute $P\left(\sim E_{1}\right)$. This is the event that node $r_{i, 1}$ has an SINR lower than $\rho_{0}$

$$
\begin{aligned}
P\left(\sim E_{1}\right) & =P\left(\rho_{r_{i, 1}} \leq \rho_{0}\right) \\
& =P\left(\frac{P \gamma_{s_{i}, r_{i, 1}}}{\sigma^{2}+P \sum_{j \neq i} \gamma_{s_{j}, r_{i, 1}}} \leq \rho_{0}\right) \\
& =P\left(\sum_{j \neq i} \gamma_{s_{j}, r_{i, 1}} \geq \frac{P \gamma_{s_{i}, r_{i, 1}}-\rho_{0} \sigma^{2}}{P \rho_{0}}\right) \\
& \leq P\left(\sum_{j \neq i} \gamma_{s_{j}, r_{i, 1}} \geq \frac{P \beta_{n}-\rho_{0} \sigma^{2}}{P \rho_{0}}\right) \\
& =P\left(\frac{1}{k-1} \sum_{j \neq i} \gamma_{s_{j}, r_{i, 1}}-\mu_{\gamma} \geq \frac{P \beta_{n}-\rho_{0} \sigma^{2}}{(k-1) P \rho_{0}}-\mu_{\gamma}\right) \\
& \leq P\left(\left|\frac{1}{k-1} \sum_{j \neq i} \gamma_{s_{j}, r_{i, 1}}-\mu_{\gamma}\right| \geq \frac{P \beta_{n}-\rho_{0} \sigma^{2}}{(k-1) P \rho_{0}}-\mu_{\gamma}\right) \\
& \leq \frac{\sigma_{\gamma}^{2} /(k-1)}{\left(\frac{P \beta_{n}-\rho_{0} \sigma^{2}}{(k-1) P \rho_{0}}-\mu_{\gamma}\right)^{2}}
\end{aligned}
$$

where the first inequality is because $\gamma_{s_{i}, r_{i, 1}} \geq \beta_{n}$ and (8) comes from the Chebyshev inequality and the fact that the variance of $\frac{1}{k-1} \sum_{j \neq i} \gamma_{s_{j}, r_{i, 1}}$ is $\sigma_{\gamma}^{2} /(k-1)$. The second inequality requires the condition $\frac{P \beta_{n}-\rho_{0} \sigma^{2}}{(k-1) P \rho_{0}}-\mu_{\gamma} \geq 0$, or

$$
\rho_{0} \leq \frac{\beta_{n}}{\frac{\sigma^{2}}{P}+(k-1) \mu_{\gamma}} .
$$

This condition on $\rho_{0}$ is intuitively satisfying: if we assume that $k$ is large, then we expect the interference term in the denominator of the SINR to be approximately $(k-1) \mu_{\gamma}$. This would imply that setting the threshold $\rho_{0}$ to less than $\frac{\beta_{n}}{\frac{\sigma^{2}}{P}+(k-1) \mu_{\gamma}}$ would be sufficient to ensure that most hops would exceed this threshold.

Note that in the previous analysis for $P\left(\rho_{r_{i}, t} \leq \rho_{0}\right)$, we have assumed that there are $(k-1)$ interference terms. This would be true if all $k$ messages are being transmitted in that particular time slot. However, this may not be the case, if, by that time slot, some of the messages have already reached their destinations successfully or have already failed to be decoded at some at some relay node. In such a case, there will be fewer than $(k-1)$ interference terms. This means that the calculation above is conservative and the actual probability of error may be smaller than that obtained above. However, from the relevant theory involving random graphs as well as from the simulations, we expect the path lengths to cluster quite densely rather than taking on a wide range of values. Thus, most messages reach their destination within very few time slots of each other. Therefore, we believe that the above error analysis is not too conservative and hence do not expect a significantly lower error probability in practice.

We define $\epsilon_{n}$ to be the probability that the SINR threshold is not exceeded along one or more of the hops. From (7), $\epsilon_{n} \leq$ 
$h P\left(\sim E_{1}\right)$. We force $h P\left(\sim E_{1}\right)$ to go to zero. From Lemma 1, $h$ is at most $\frac{\log n}{\alpha \log n p}$ and we have

$$
\epsilon_{n} \leq h P\left(\sim E_{1}\right) \leq \frac{\log n}{\alpha \log n p} \frac{\sigma_{\gamma}^{2}}{(k-1)\left(\frac{P \beta_{n}-\rho_{0} \sigma^{2}}{(k-1) P \rho_{0}}-\mu_{\gamma}\right)^{2}}
$$

and we require the right-hand side to go to zero.

We mention that inequality (10) requires $\gamma$ to have a variance that does not go to infinity. There are several distributions of practical interest in which the variance does go to infinity, but the mean is finite. (For example, $f(\gamma)=\frac{c}{(1+\gamma)^{m}}$ for $m>2$ is considered in [28].) In this case, an alternative inequality can be obtained by applying the Markov bound to $P\left(\sim E_{1}\right)$ rather than the Chebyshev bound. The result is

$$
\epsilon_{n} \leq h P\left(\sim E_{1}\right) \leq \frac{\log n}{\alpha \log n p} \frac{(k-1) \mu_{\gamma} P \rho_{0}}{P \beta_{n}-\rho_{0} \sigma^{2}} .
$$

An achievable throughput can be obtained using either the Chebyshev bound of (10) or the Markov inequality above. Theorem 1 is obtained using the Chebyshev inequality. Theorem 4, presented at the end of Section VII, is an achievability result obtained using the Markov inequality (11). In general, we expect the Chebyshev inequality to be tighter than the Markov inequality and therefore prefer to use Theorem 1 whenever $\gamma$ has finite variance.

\section{Proof OF THEOREM 1}

We now combine the results of Section V on the maximum number of noncolliding paths and Section VI on the probability of successful transmission along these paths. We need $p=P\left(\gamma \geq \beta_{n}\right)=Q_{n}\left(\beta_{n}\right)=\frac{\log n+\omega_{n}}{n}$ in order to do scheduling. In addition, we need

1) to have noncolliding paths (Theorem 3 )

$$
k \leq \alpha n \frac{\log n p}{\log n}
$$

2) to meet the SINR threshold [(10)]

$$
\epsilon_{n} \leq \frac{\log n}{\alpha \log n p} \frac{\sigma_{\gamma}^{2}}{(k-1)\left(\frac{P \beta_{n}-\rho_{0} \sigma^{2}}{(k-1) P \rho_{0}}-\mu_{\gamma}\right)^{2}} \rightarrow 0 ;
$$

3) to apply the Chebyshev inequality [(9)]

$$
\rho_{0} \leq \frac{\beta_{n}}{\frac{\sigma^{2}}{P}+(k-1) \mu_{\gamma}} .
$$

To satisfy condition 3 ), we set

$$
\rho_{0}=\frac{a_{n} \beta_{n}}{\frac{\sigma^{2}}{P}+(k-1) \mu_{\gamma}}
$$

where $0 \leq a_{n} \leq 1$. Substituting for this in the second condition, we get

$$
\begin{aligned}
\epsilon_{n} & \leq \frac{a_{n}^{2}}{\alpha\left(1-a_{n}\right)^{2}} \frac{\left(k_{n}\left(\beta_{n}\right)-1\right) \sigma_{\gamma}^{2}}{\left(\frac{\sigma^{2}}{P}+\left(k_{n}\left(\beta_{n}\right)-1\right) \mu_{\gamma}\right)^{2}} \frac{\log n}{\log \left(n Q_{n}\left(\beta_{n}\right)\right)} \\
& \rightarrow 0 .
\end{aligned}
$$

This and condition 1) are the only conditions on $k$. For any $k$ satisfying these two conditions, we get an achievable throughput. This gives us Theorem 1 .

The theorem gives an achievable throughput as a function of $\beta_{n}, a_{n}$ and $k_{n}$ but does not attempt to optimize these parameters. Because $\epsilon_{n}$ goes to zero and $h$ is determined by $\beta_{n}$, to find the optimum $k$ we need to maximize $k \log \left(1+\rho_{0}\right)=$ $k \log \left(1+\frac{a_{n} \beta_{n}}{\frac{\sigma^{2}}{P}+(k-1) \mu_{\gamma}}\right)$ over $k$. In the particular case when $\frac{\sigma^{2}}{P}-\mu_{\gamma}$ is positive, the expression is nondecreasing in $k$ (the first derivative is nonnegative). Hence, satisfying (5) with equality is optimum. This proves Corollary 1.

Finally, we state without proof an achievability result obtained using the Markov inequality (11) to bound the error, rather than the Chebyshev inequality (10). This result can be used in place of Theorem 1 for distributions that have a finite mean but an infinite variance.

Theorem 4: Consider a network on $n$ nodes whose edge strengths are drawn i.i.d. from a probability distribution function $f_{n}(\gamma)$. Let $F_{n}(\gamma)$ denote the cumulative distribution function corresponding to $f_{n}(\gamma)$ and define $Q_{n}(\gamma)=1-F_{n}(\gamma)$. Choose any $\beta_{n}$ such that $Q_{n}\left(\beta_{n}\right)=\frac{\log n+\omega_{n}}{n}$, where $\omega_{n} \rightarrow \infty$ as $n \rightarrow \infty$. Then, there exists a positive constant $\alpha$ such that a throughput of

$$
\begin{aligned}
T=\left(1-\epsilon_{n}\right) \alpha & k_{n}\left(\beta_{n}\right) \frac{\log \left(n Q_{n}\left(\beta_{n}\right)\right)}{\log n} \\
& \times \log \left(1+\frac{\beta_{n}}{\frac{\sigma^{2}}{P}+b_{n}\left(k_{n}\left(\beta_{n}\right)-1\right) \mu_{\gamma}}\right)
\end{aligned}
$$

is achievable for any positive $b_{n}$ such that $b_{n} \geq 1$ and any $k_{n}\left(\beta_{n}\right)$ that satisfy the following conditions:

1)

$$
k_{n}\left(\beta_{n}\right) \leq \alpha n \frac{\log \left(n Q_{n}\left(\beta_{n}\right)\right)}{\log n}
$$

2)

$$
\epsilon_{n} \leq \frac{1}{\alpha b_{n}} \frac{\log n}{\log \left(n Q_{n}\left(\beta_{n}\right)\right)} \rightarrow 0 ;
$$

where $\mu_{\gamma}$ is the mean of $\gamma$. The SINR threshold is $\rho_{0}=$ $\frac{\beta_{n}}{P}+b_{n}\left(k_{n}\left(\beta_{n}\right)-1\right) \mu_{\gamma}$.

\section{EXAMPLES AND APPLICATIONS}

In this section, we apply Theorem 1 to some particular channel distributions. Since, as in geometric models, the throughput is often interference limited, we find that densities that lead to significant interference per transmitter generally underperform those that generate only a small amount of interference. 


\section{A. Shadow Fading Model}

We revisit the model (1)

$$
f_{n}(\gamma)=\left(1-p_{n}\right) \delta(\gamma)+p_{n} \delta(\gamma-1)
$$

where $\delta(\cdot)$ is the Dirac delta-function. This pdf models the situation where strong shadow fading is present. The signal power is 0 in the presence of an obstruction and is 1 , otherwise. We find the value of $p$ that maximizes the throughput. (We drop the subscript $n$.) A natural choice for the goodness threshold $\beta_{n}$ is 1 , which gives $Q(\beta)=p$. We need to satisfy $p \geq\left(\log n+\omega_{n}\right) / n$ (where $\omega_{n} \rightarrow \infty$ ) in order to use Theorem 1 .

Note that we have $\mu_{\gamma}=p$ and $\sigma_{\gamma}^{2}=p(1-p)$. It is possible to check that unless $p \rightarrow 0$, the throughput is at most constant. With $p \rightarrow 0$ and sufficiently large $n$, the condition $\frac{\sigma^{2}}{P}-\mu_{\gamma}=$ $\frac{\sigma^{2}}{P}-p \geq 0$ is satisfied. Therefore, according to Corollary 1 the maximum possible $k$ achieves maximum throughput. Hence, we consider $k=\alpha n \frac{\log n p}{\log n}$. Since $p=\frac{\log n+\omega_{n}}{n}, k \rightarrow \infty$ and we may replace $k-1$ by $k$ in (6) and the SINR threshold. Since $k p$ also goes to infinity, (6) becomes

$$
\epsilon_{n} \leq \frac{a_{n}^{2}}{\alpha^{2}\left(1-a_{n}\right)^{2}} \frac{\log ^{2} n}{\log ^{2}(n p)} \frac{1}{n} \rightarrow 0
$$

. Therefore, $a_{n}$ may be any positive constant $a<1$. With this, the SINR threshold becomes

$$
\rho_{0}=\frac{a}{\frac{\sigma^{2}}{P}+\alpha n p \frac{\log n p}{\log n}} \approx \frac{a}{\alpha n p \frac{\log n p}{\log n}}
$$

which goes to zero. Thus, $\log \left(1+\rho_{0}\right) \approx \rho_{0}$ and we have $\frac{k}{h} \log \left(1+\rho_{0}\right)=\frac{a \alpha}{p} \frac{\log n p}{\log n}$. This is maximized when $p$ is as small as possible, or $p=\frac{\log n+\omega_{n}}{n}$. The result is summarized in Corollary 2.

Corollary 2: Consider a network on $n$ nodes where edge strengths are drawn i.i.d. from the distribution in (15). Then, for large $n$ the throughput is maximized for $p=\frac{\log n+\omega_{n}}{n}$ and is given by

$$
\begin{aligned}
T=\left(1-\frac{a^{2}}{\alpha^{2}(1-a)^{2}} \frac{\log ^{2} n}{\log ^{2}\left(\log n+\omega_{n}\right)} \frac{1}{n}\right) \\
\quad \times a \alpha \frac{\log \left(\log n+\omega_{n}\right)}{\left(\log n+\omega_{n}\right) \log n} n
\end{aligned}
$$

as $n \rightarrow \infty$, where $\omega_{n}$ is any function going to infinity and $0<a<1$ and $\alpha<1$ are constants.

This throughput is almost linear in $n$ and requires the network to be sparsely connected; with a connection probability of $(\log n) / n$, each node is connected with only approximately $\log n$ other nodes. For example, with $n=1000$ nodes, we have $(\log n) / n=0.0069$ and each node connects on average to only seven other nodes. Perhaps surprisingly, increasing or decreasing this connectivity has a detrimental effect. While it is clear that it is possible for a network to be under-connected, it is apparently also possible for a network to be over-connected. The simulations in Section X-B also demonstrate this effect.

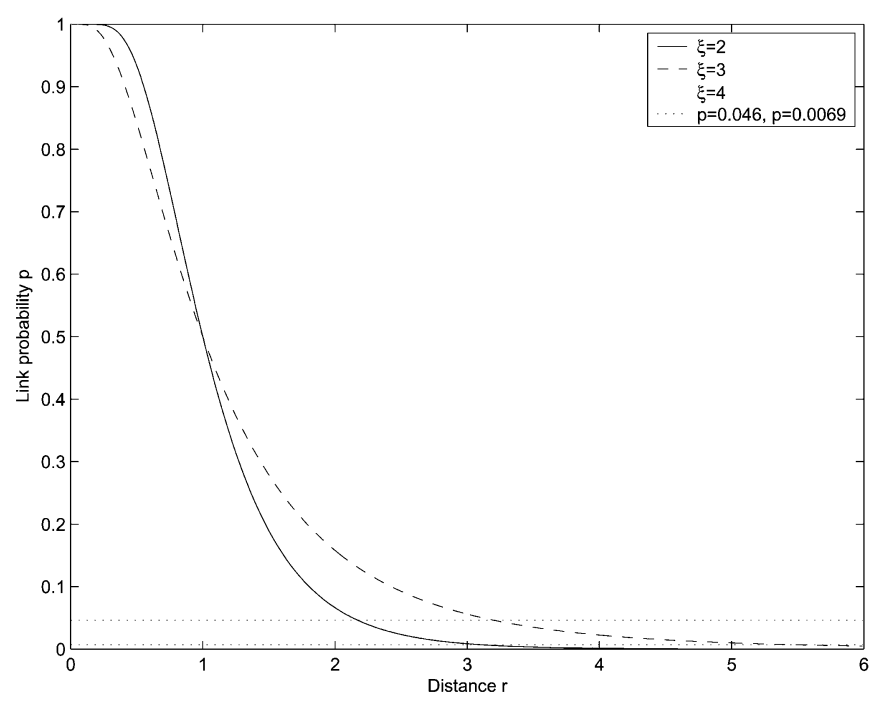

Fig. 3. Link probability $p$ versus distance $\hat{r}$ as given by (17) for $\xi=2,3,4$. Also shown are dotted lines at $p=(\log 100) / 100 \approx 0.046$ and $p=(\log 1000) / 1000 \approx 0.0069$ indicating the optimum throughput point for shadow-fading with 100 and 1000 nodes respectively. As a function of $\hat{r}, p$ is relatively insensitive for large $\hat{r}$.

1) Implications for a Certain Radio Model: In [7] and [8], a wireless connectivity model is introduced where the probability of a good link is expressed as

$$
p(\hat{r})=\frac{1}{2}\left[1-\operatorname{erf}\left(3.07 \frac{\log \hat{r}}{\xi}\right)\right]
$$

where $\hat{r}$ is a (suitably normalized) distance between the transmitter and receiver and $\xi$ is a parameter that depends on the degree of shadow fading and the distance pathloss exponent. Usually $\xi \in[0,6]$ where large values indicate a strong shadow component. The links between different sources or destinations are modeled as statistically independent.

For nodes approximately $\hat{r}$ from each other, the model (16) is equivalent to our model of shadow fading (15) with $p=p(\hat{r})$. As we show in Section VIII-A, maximum throughput is attained for $p \approx(\log n) / n$. The "equivalent distance" for nodes is found by solving

$$
p=\frac{\log n}{n}=\frac{1}{2}\left[1-\operatorname{erf}\left(3.07 \frac{\log \hat{r}}{\xi}\right)\right]
$$

for $\hat{r}$. Nodes approximately this distance from each other then have the excellent throughput promised in Corollary 2. Because we cannot have a large network of nodes exactly equidistant from each other, (17) only has operational meaning if the link probability is relatively insensitive to the distance $\hat{r}$ when $p \approx$ $(\log n) / n$. We show that it is.

As the number of nodes $n$ increases, the optimum link-probability $(\log n) / n$ decreases or, equivalently, the distance $\hat{r}$ between nodes increases. For large $\hat{r}$, we may approximate $\frac{1}{2}(1-$ $\operatorname{erf} x) \approx 1 /(2 \sqrt{\pi} x) \exp \left(-x^{2}\right)$, and thus (17) becomes

$$
p=\frac{\log n}{n}=\frac{\xi}{10.88 \log \hat{r}} e^{-3.07 \log ^{2} \hat{r} / \xi} .
$$

The sensitivity of $p$ as a function of $\hat{r}$ is very low when $p$ is small. We show this in Fig. 3, where we display $p$ versus $\hat{r}$ for various values of $\xi$. The dotted lines in the figure shows the approximate optimum operating point $p$ for networks with 100 and 
1000 nodes. We see that the optimum $p$ is generally very small and relatively insensitive to $\hat{r}$, and the best network performance is generally therefore obtained when the nodes are relatively far apart from one another, with a wide range of acceptable distances. This suggests that a large high-throughput network of nodes with optimum (small) $p$ is possible.

We comment that the authors in [8] also consider how shadow fading can reduce the hop-count in a network and they use some graph-theoretic concepts in their arguments. They do not, however, attempt to obtain a throughput result by finding simultaneous noncolliding paths, nor do they incorporate the detrimental effects of interference to show that a network can be "too connected."

\section{B. Density Obtained From a Decay Law}

In this example, we construct a pdf from the marginal density of the channel strengths in a geometric model. For every node, the channel coefficients to the remaining nodes follow a deterministic law based on distance. If we group these coefficients according to their magnitude $\gamma$, we obtain a certain number of coefficients whose magnitude falls in the interval $(\gamma, \gamma+d \gamma)$. We seek a pdf whose average number of magnitudes matches this deterministic law.

In an actual geometric model, the distribution of channel magnitudes depends on the location of the nodes. We make a simplifying assumption: We suppose that the nodes are in a circular disk and consider the node at the center of the disk to derive the density. We thereby ignore the effects of the disk boundary. We assume the nodes are dropped with density $\Delta$ (nodes per unit area) but ensuring a minimum distance of $d$ from the center. The area of the entire disk is $n / \Delta$.

In deriving the density of the channel coefficients, we use a power law of the form $g(r)$, where a node transmitting with power $P$ is received by another node at distance $r$ with power $\mathrm{Pg}(r)$. We assume that $g(\cdot)$ is monotonically decreasing. The most significant difference between our model and the standard geometric model is in the independence of the channel coefficients in our model that does not exist in the geometric model. The geometric model has a correlation structure in the coefficients where channels of similar strength are clustered in rings around the center node. In our model, coefficients of similar strength, although the same in number as the geometric model, are distributed randomly and not necessarily geometrically colocated.

Consider a node at the center of the disk transmitting at power $P$. The fraction of nodes receiving power $\leq \gamma P$ is given by $1-\frac{\Delta}{n} 2 \pi\left(\left(g^{-1}(\gamma)\right)^{2}-d^{2}\right)$ where

$$
\gamma \in\left[g\left(\sqrt{\frac{n}{2 \pi \Delta}+d^{2}}\right), g(d)\right]
$$

In particular, if we have a decay law of the form $g(r)=\frac{1}{r^{m}}$, this tells us that the fraction of nodes receiving power $\leq \gamma P$ is given by

$$
1-\frac{\Delta}{n} 2 \pi\left(\frac{1}{\gamma^{2 / m}}-d^{2}\right)
$$

for

$$
\gamma \in\left[\left(\frac{2 \pi \Delta}{n+2 \pi \Delta d^{2}}\right)^{m / 2}, \frac{1}{d^{m}}\right] .
$$

This is a cumulative distribution function and by differentiating it with respect to $\gamma$ we obtain the pdf for the edge strengths seen by the central node as

$$
\begin{aligned}
f_{n}(\gamma) & =\frac{4 \pi \Delta}{n m} \frac{1}{\gamma^{1+\frac{2}{m}}}, \\
\gamma & \in\left[\left(\frac{2 \pi \Delta}{n+2 \pi \Delta d^{2}}\right)^{m / 2}, \frac{1}{d^{m}}\right], \quad m>0 .
\end{aligned}
$$

We assume that connections are drawn i.i.d. from this distribution.

We apply our results to this network and obtain the following corollary.

Corollary 3: Consider a network on $n$ nodes where edge strengths are drawn i.i.d. from the distribution

$$
\begin{aligned}
f_{n}(\gamma) & =\frac{4 \pi \Delta}{n m} \frac{1}{\gamma^{1+\frac{2}{m}}}, \\
\gamma & \in\left[\left(\frac{2 \pi \Delta}{n+2 \pi \Delta d^{2}}\right)^{m / 2}, \frac{1}{d^{m}}\right], \quad m>0 .
\end{aligned}
$$

Then, the achievable values of $\epsilon_{n}$ and throughputs are achievable:

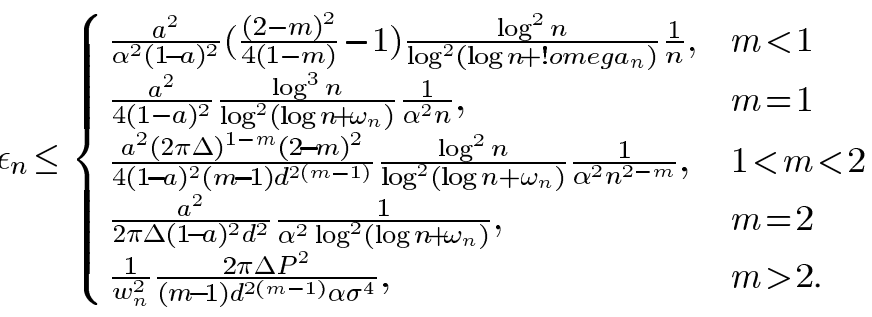

$$
\begin{aligned}
& T= \begin{cases}\left(1-\epsilon_{n}\right) \frac{a(2-m) \alpha}{2} \frac{\log \left(\log n+\omega_{n}\right)}{\log n\left(\log n+\omega_{n}\right)^{m / 2}} n^{m / 2}, & m<1 \\
\left(1-\epsilon_{n}\right) \frac{a \alpha}{2} \frac{\log \left(\log n+\omega_{n}\right)}{\log n\left(\log n+\omega_{n}\right)^{1 / 2}} n^{1 / 2}, & m=1 \\
\left(1-\epsilon_{n}\right) \frac{a(2-m) \alpha}{2} \frac{\log \left(\log n+\omega_{n}\right)}{\log n\left(\log n+\omega_{n}\right)^{m / 2}} n^{m / 2}, & 1<m<2 \\
\left(1-\epsilon_{n}\right) a \alpha \frac{\log \left(\log n+\omega_{n}\right)}{\log n\left(\log n+\omega_{n}\right)} n, & m=2 \\
\left(1-\epsilon_{n}\right) \frac{P \alpha^{2}(2 \pi \Delta)^{m / 2}}{\sigma^{2} w_{n}} \frac{\log ^{2}\left(\log n+\omega_{n}\right)}{\log ^{2} n\left(\log n+\omega_{n}\right)^{m / 2}} n, & m>2\end{cases}
\end{aligned}
$$

where $a<1$ and $\alpha<1$ are constants and $\omega_{n}$ and $w_{n}$ are functions going to infinity.

We see that almost linear throughput can be obtained for $m \geq 2$. This differs substantially from the $O(\sqrt{n})$ or $O(\sqrt{n / \log n})$ results obtained for the structured deterministic model with the same decay law. Our results show that it is not the marginal distribution of the power that impedes the throughput in a geometric power-decay network, but rather the spatial distribution of these powers. We notice that in the 
geometric model, nodes transmit to their nearest neighbors and therefore messages take up to $\sqrt{n}$ hops to reach their intended destinations. In the random model, nodes talk across their good links and only $\log n$ hops are necessary to send a message across. This is due to two factors: The first is that far fewer nodes get drowned out in the interference when one node transmits, thus permitting more nodes to transmit simultaneously and the second is that any two nodes in the random graph are only $\log n$ hops away, rather than the $\sqrt{n}$ of the deterministic model.

It is easy to see that both advantages come about from the absence of the geometric constraints compared to the deterministic model. If we think of the example of this section in terms of the network of [11], but with the spatial constraints removed, it is not surprising that the throughput scaling is much more favorable than $O(\sqrt{n / \log n})$. We can conclude that this improvement arises from the introduction of randomness in the network model.

\section{A Distribution With Constant Mean and Variance}

Consider a general distribution $f_{n}(\gamma)$ that has a constant mean and variance. For such a distribution, one can show that choosing $k \rightarrow \infty$ is the best choice and leads to the following corollary.

Corollary 4: Consider a network on $n$ nodes where edge strengths are drawn i.i.d. from a distribution $f_{n}(\gamma)$ where the mean $\mu_{\gamma}$ and variance $\sigma_{\gamma}^{2}$ of $\gamma$ are independent of $n$. Then, the throughput is given by

$$
\begin{aligned}
T=\left(1-\frac{a^{2} \sigma_{\gamma}^{2}}{\alpha^{2}(1-a)^{2} \mu_{\gamma}^{2}} \frac{\log ^{2} n}{\log ^{2}\left(n Q_{n}\left(\beta_{n}\right)\right)} \frac{1}{n}\right) \\
\times \frac{a \alpha}{\mu_{\gamma}} \frac{\beta_{n} \log \left(n Q_{n}\left(\beta_{n}\right)\right)}{\log n}
\end{aligned}
$$

and the optimum $\beta_{n}$ maximizes $\beta_{n} \log \left(n Q_{n}\left(\beta_{n}\right)\right)$ while satisfying $Q_{n}\left(\beta_{n}\right) \geq \frac{\log n+\omega_{n}}{n}$.

Perhaps surprisingly, distributions with constant mean and variance, while allowing us to apply Corollary 4, can have widely different throughputs. This is illustrated by the next few examples.

\section{An Exponential Density}

Let $f_{n}(\gamma)=e^{-\gamma}$. For this pdf, the mean and variance are constant, and we can apply Corollary 4 . The obtained througput is summarized later.

Corollary 5: Consider a network on $n$ nodes where edge strengths are drawn i.i.d. from a distribution $f_{n}(\gamma)=e^{-\gamma}$. Then, a throughput of

$$
T=\left(1-\frac{a^{2}}{\alpha^{2}(1-a)^{2}} \frac{4}{n}\right) \frac{a \alpha \log n}{4}
$$

is achievable as $n \rightarrow \infty$ where $\alpha<1, a<1$ are constants.

We see that a random network dominated by an exponential pdf has a throughput that grows only logarithmically with $n$. This network has good connectivity since the number of hops is small, but is also unfortunately dominated by interference. Thus, only few transmissions can occur simultaneously. We show in Section IX that this throughput is tight to first order in $n$.

\section{E. A Heavy-Tail Distribution}

Consider a network on $n$ nodes where edge strengths are drawn i.i.d. from $f_{n}(\gamma)=\frac{c}{1+\gamma^{4}}, \gamma \geq 0$ where $c$ is such that $f_{n}(\gamma)$ integrates to 1 . Clearly, the mean and variance of this distribution are constant with respect to $n$. Therefore, we can apply Corollary 4 . The optimum $\beta_{n}$ turns out to be $\frac{n^{1 / 3}}{\left(\log n+\omega_{n}\right)^{1 / 3}} \frac{c^{1 / 3}}{3^{1 / 3}}$ and we have the following corollary.

Corollary 6: Consider a network on $n$ nodes where edge strengths are drawn i.i.d. from the distribution $f_{n}(\gamma)=\frac{c}{1+\gamma^{4}}$, $\gamma \geq 0$. The throughput is then

$$
\begin{aligned}
T= & \left(1-\frac{a^{2} \sigma_{\gamma}^{2}}{\alpha^{2} \mu_{\gamma}^{2}(1-a)^{2}} \frac{\log ^{2} n}{\log ^{2}\left(\log n+\omega_{n}\right)} \frac{1}{n}\right) \\
& \times \frac{a(c / 3)^{1 / 3} \alpha}{\mu_{\gamma}} \frac{\log \left(\log n+\omega_{n}\right)}{\log n\left(\log n+\omega_{n}\right)^{1 / 3}} n^{1 / 3} \\
\approx & \frac{a(c / 3)^{1 / 3} \alpha}{\mu_{\gamma}} \frac{\log \log n}{\log ^{4 / 3} n} n^{1 / 3} .
\end{aligned}
$$

\section{F. Lognormal Fading}

Consider a network on $n$ nodes where edge strengths are drawn from a lognormal distribution. Thus $f_{n}(\gamma)=$ $\frac{1}{S \sqrt{2 \pi} \gamma} \exp \left(-(\log \gamma-M)^{2} / 2 S^{2}\right), \gamma \geq 0$ where $M$ and $S$ are parameters of the distribution. We have $\mu_{\gamma}=e^{M+S^{2} / 2}$ and $\sigma_{\gamma}^{2}=e^{S^{2}+2 M}\left(e^{S^{2}}-1\right)$. Because the mean and variance are constant, we may apply Corollary 4 and get the following result.

Corollary 7: Consider a network on $n$ nodes where edge strengths are drawn i.i.d. from the distribution

$$
f_{n}(\gamma)=\frac{1}{S \sqrt{2 \pi} \gamma} \exp \left(-(\log \gamma-M)^{2} / 2 S^{2}\right), \quad \gamma \geq 0
$$

The throughput is then

$$
\begin{aligned}
T= & \left(1-\frac{a^{2} \sigma_{\gamma}^{2}}{\alpha^{2} \mu_{\gamma}^{2}(1-a)^{2}} \frac{\log ^{2} n}{\log ^{2}\left(\log n+\omega_{n}\right)} \frac{1}{n}\right) \\
& \times \frac{a \alpha}{\mu_{\gamma}} \frac{e^{M} e^{\sqrt{2} S \sqrt{\log n}} \log \left(\log n+\omega_{n}\right)}{\log n} \\
\approx & \frac{a \alpha e^{M+\sqrt{2} S}}{\mu_{\gamma}} \frac{\log \log n}{\log n} e^{\sqrt{\log n}} .
\end{aligned}
$$

We see that the throughput grows as $e^{\sqrt{\log n}}$ which can also

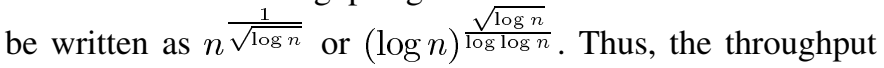
is considerably better than $\log n$ obtained with the exponential density (Rayleigh fading).

\section{G. Tradeoff Between $k$ and $\rho_{0}$}

In most of the previous examples, we notice that the optimum $k$ goes to infinity; hence the optimum $\rho_{0}=\frac{a \beta_{n}}{\frac{\sigma^{2}}{P}+(k-1) \mu_{\gamma}}$ goes to zero. In these cases we approximate $\log \left(1+\rho_{0}\right)$ by $\rho_{0}$. In addition, if $k \mu_{\gamma}$ goes to infinity, we can further approximate $\rho_{0}$ as $\frac{a \beta_{n}}{k \mu_{\gamma}}$. In this case, we have $\frac{k}{h} \log \left(1+\rho_{0}\right) \approx \frac{a \beta_{n}}{h \mu_{\gamma}}$. This expression depends only on $\beta_{n}$ and is independent of $k$ and $\rho_{0}$. We can therefore increase (decrease) $k$, thus decreasing (increasing) 
$\rho_{0}=\frac{a \beta_{n}}{\frac{\sigma^{2}}{P}+(k-1) \mu_{\gamma}}$ and (as long as $\left.k \mu_{\gamma} \rightarrow \infty\right)$ the throughput remains unaffected. Hence, it is sometimes possible to tradeoff the number of simultaneously communicating source-destination pairs with the SINRs at which they communicate without affecting the aggregate throughput.

\section{UPPER BOUNDS}

Our method of finding the throughput relies on finding good edges along which the desired communication can take place. This method does not preclude other methods from possibly doing better. In the cases where the throughput is of the form $\frac{n}{\log ^{d} n}$, the optimal throughput cannot be better by more than the factor $\log ^{d} n$ because the maximum throughput cannot scale more than linearly (unless the channel density is somehow chosen such that the maximum received power increases as the number of nodes increases-we exclude such densities here).

However, when the throughput we compute turns out to be of the order of $n^{d}$ for $d<1$, or $\log n$ as with the exponential density, it is not clear that we cannot do better. In this section, we present an approach to computing an upperbound on throughput that shows that we sometimes cannot do better.

The throughput is given by $(1-\epsilon) \frac{k}{h} \log \left(1+\rho_{0}\right)$. We ignore the $h$ in the denominator and find an upperbound for $k \log \left(1+\rho_{0}\right)$. Thus, we allow ourselves to choose $k$ source-destination pairs from a given network and find the highest SINR threshold that can be met for all of them simultaneously. This is equivalent to finding a bound for the best single hop communication. Clearly, by doing this, our achievability results are certain to be at least a factor of $h$ away from the upperbound. However, we know that $h$ can be no larger than $\frac{\log n}{\log \left(\log n+\omega_{n}\right)}$, which is often a small factor.

There are $\left(\begin{array}{l}n \\ k\end{array}\right)\left(\begin{array}{c}n-k \\ k\end{array}\right) k$ ! ways of choosing $k$ source-destination pairs in a network. Assume that a threshold $\rho_{0}$ is fixed. Then, for a randomly drawn set of source-destination pairs, there is a probability, say $p_{s}$, that a received message satisfies the SINR threshold and is decoded successfully. The probability that all $k$ received messages satisfy the threshold is $p_{s}^{k}$. Therefore, for a given pair $\left(k, \rho_{0}\right)$, the expected number of sets of $k$ source-destination pairs that satisfy the threshold $\rho_{0}$ is

$$
M_{n}\left(k, \rho_{0}\right)=\left(\begin{array}{l}
n \\
k
\end{array}\right)\left(\begin{array}{c}
n-k \\
k
\end{array}\right) k ! p_{s}^{k} .
$$

Note that $p_{s}$ depends on $\rho_{0}, k$ and the pdf $f_{n}(\gamma)$ from which the connections are drawn. We say that a $\left(k, \rho_{0}\right)$ pair is feasible if there exists at least one set of $k$ source-destination pairs such that each of the $k$ SINRs exceeds $\rho_{0}$. The probability that a particular $\left(k, \rho_{0}\right)$.pair is feasible can be bounded as follows:

$P\left(\left(k, \rho_{0}\right)\right.$ is feasible $)$

$$
\begin{aligned}
& =P\left(\# \text { of } k \text {-pairs that satisfy the threshold } \rho_{0} \text { is } \geq 1\right) \\
& \leq E\left(\# k \text {-pairs that satisfy the threshold } \rho_{0}\right) \\
& =M_{n}\left(k, \rho_{0}\right)
\end{aligned}
$$

where the Markov inequality is used.

If for a particular choice of $\left(k, \rho_{0}\right)$ we have $M_{n}\left(k, \rho_{0}\right)$ going to zero then that choice is infeasible. Otherwise $\left(k, \rho_{0}\right)$ may be feasible. We can thereby characterize all $\left(k, \rho_{0}\right)$ pairs that may be feasible. The largest value of $k \log \left(1+\rho_{0}\right)$ taken over these pairs gives us an upperbound on the throughput.
Note that this approach is general and can be used for any pdf, but requires a calculation of

$$
p_{s}=P\left(\frac{P \gamma_{1}}{\sigma^{2}+P \sum_{i=2}^{k} \gamma_{i}} \geq \rho_{0}\right) .
$$

where all the channel coefficients in the SINR expression are drawn i.i.d. according to $f_{n}(\gamma)$. For certain densities, such as the exponential, we may compute $p_{s}$ and get an upperbound as follows.

$$
\begin{aligned}
& \text { If } f_{n}(\gamma)=e^{-\gamma} \text {, then } \\
& \qquad p_{s}=P\left(\frac{P \gamma_{1}}{\sigma^{2}+P \sum_{i=2}^{k} \gamma_{i}} \geq \rho_{0}\right)=\frac{e^{-\frac{\sigma^{2}}{P} \rho_{0}}}{\left(1+\rho_{0}\right)^{k-1}} .
\end{aligned}
$$

With this

$$
M_{n}\left(k, \rho_{0}\right)=\left(\begin{array}{l}
n \\
k
\end{array}\right)\left(\begin{array}{c}
n-k \\
k
\end{array}\right) k ! \frac{e^{-\frac{\sigma^{2}}{P} k \rho_{0}}}{\left(1+\rho_{0}\right)^{k(k-1)}} .
$$

We now want to characterize $\left(k, \rho_{0}\right)$ pairs for which $M_{n}\left(k, \rho_{0}\right)$ does not go to zero. We have

$$
\begin{aligned}
M_{n}\left(k, \rho_{0}\right) & =\frac{n !}{(n-2 k) ! k !} p_{s}^{k} \leq \frac{n !}{(n-2 k) !} p_{s}^{k} \\
& \leq n^{2 k} \frac{e^{-\frac{\sigma^{2}}{P} k \rho_{0}}}{\left(1+\rho_{0}\right)^{k(k-1)}} \\
& \leq\left(n^{2} \frac{1}{\left(1+\rho_{0}\right)^{k}}\right)^{k}=e^{k\left(2 \log n-k \log \left(1+\rho_{0}\right)\right)} .
\end{aligned}
$$

If $k$ goes to infinity (with $n$ ) and $2 \log n-k \log \left(1+\rho_{0}\right)$ is negative then $M_{n}\left(k, \rho_{0}\right)$ goes to zero. Therefore, for $k$ going to infinity, we have $k \log \left(1+\rho_{0}\right) \leq 2 \log n$ as a bound on the throughput. If $k$ is constant, it is easy to see that $1+\rho_{0}$ cannot grow faster than $n^{2}$, hence the throughput is again limited by $k \log n^{2}=2 k \log n$ where $k$ is now a constant. Thus we have shown an upperbound of $c \log n$ on the throughput. This happens to coincide (to within a constant) with the throughput obtained in our achievability result (Section VIII-D). In our scheme it turns out that using two hops is optimal for any $n$. Hence, although the upperbound derived here is on $k \log \left(1+\rho_{0}\right)$, it matches the achievability result for $\frac{k}{h} \log \left(1+\rho_{0}\right)$ very closely.

\section{Simulations AND CONCLUSION}

Theorem 1 gives a very specific achievability result but (4) involves a constant $\alpha$ that is not explicit. This constant has its origins in Theorem 3 where the number of vertex-disjoint paths is computed. When we are confronted with a specific network with a finite number of nodes $n$, we would like an explicit estimate of the number of noncolliding paths. In this section we provide such an estimate; we also briefly introduce the notion of "bad" edges, discuss decentralized algorithms for attaining our achievability results, and provide computer simulations of some of the networks analyzed in Section VIII. 


\section{A. Noncolliding Paths}

In Section V, we use a result of [19] to establish the existence of noncolliding paths. In this section, we present a constructive method of obtaining these paths and analyze the expected number of noncolliding paths thereby obtained. The algorithm we present is used extensively in Section X-B.

We begin by choosing nodes $1, \ldots, n / 2$ as source nodes and nodes $n / 2+1, \ldots, n$ as their respective destination nodes. For the first source-destination pair, a shortest path connecting them (using only links that exceed $\beta$ ) is found. This is done using a standard breadth-first search algorithm [20] in which a rooted tree is constructed. All of the nodes begin by being "undiscovered". The source node acts as the root of the tree (at depth zero) and is labeled as "discovered". We then find all the nodes that are its neighbors and call them discovered. These are at distance one from the source and hence at depth one in the breadth-first search tree. The nodes at depth one are then processed successively. All of the neighbors of each node that are still undiscovered are put in the tree at depth two and their labels are changed to discovered. The process continues till there are no undiscovered nodes. Clearly, each node appears at most once in the tree. A shortest path from the source (root) to the destination is obtained by simply finding that node in the tree and moving up the tree to the source node. If the destination does not appear in the tree it has no path to the source.

Once the shortest path for the $i$ th source-destination pair is established it is recorded and all $n$ nodes are relabeled as "undiscovered;" the entire process is repeated to find the shortest path for the $(i+1)$ st source-destination pair. This is done till paths are found for all $n / 2$ pairs.

We then eliminate colliding paths on this list, starting with the first source-destination pair. If a node used on the path between $s_{1}$ and $d_{1}$ collides with a node on some other path, we eliminate path 1 , otherwise, we keep it. We proceed in order and eliminate the $i$ th path if it collides with any of paths $i+1, i+2, \ldots, n / 2$ and keep it otherwise. Note that since we start with shortest paths, a relay never appears more than once on a particular path.

Let us bound the probability that paths $i$ and $j$ collide for $i \neq j$. Without loss of generality, we can set $i=1$ and $j=2$. We now have (21), as shown at the bottom of the page. The inequality is a standard union bound and the last equality is because the $h-1$ relay nodes on the $i$ th path are drawn uniformly at random from from the set of all nodes of the graph (excluding $s_{i}$ and $d_{i}$ ). (We assume that the algorithm that chooses the shortest path for $\left(s_{i}, d_{i}\right)$ does not use any knowledge of the previously chosen $i-1$ paths.)

Denote by $D_{i}$ the event of keeping the $i$ th path. This event comprises the intersection of the events that the $i$ th path does not collide with the $(i+1)$ st through $(n / 2)$ th paths. These $n / 2-i$ events are identical although they are not necessarily independent. However, for the purposes of an approximation we may assume they are independent and compute $P\left(D_{i}\right)$ as follows:

$$
\begin{aligned}
P\left(D_{i}\right) & \approx \prod_{j=i+1}^{n / 2} P(\text { paths } i \text { and } j \text { do not collide }) \\
& =(P(\text { paths } i \text { and } i+1 \text { do not collide }))^{n / 2-i} \\
& =(1-P(\text { paths } i \text { and } i+1 \text { collide }))^{n / 2-i} \\
& =(1-P(\text { paths } 1 \text { and } 2 \text { collide }))^{n / 2-i} \\
& \geq\left(1-\frac{3 h-1}{n-2}\right)^{n / 2-i}
\end{aligned}
$$

The inequality is a consequence of (21). We expect the inequality to be an approximate equality when $h$ is small. The expected number of successful paths is then

$$
\begin{aligned}
& \text { Expected \# noncolliding } \\
& \begin{array}{l}
=\sum_{i=1}^{n / 2} P\left(D_{i}\right) \\
\approx \sum_{i=1}^{n / 2}\left(1-\frac{3 h-1}{n-2}\right)^{n / 2-i} \\
=\frac{n-2}{3 h-1}\left(1-\left(1-\frac{3 h-1}{n-2}\right)^{n / 2}\right) \\
\approx \frac{n-2}{3 h-1}
\end{array}
\end{aligned}
$$

because $(1-x / n)^{n / 2} \approx e^{-x / 2}$ decreases rapidly with $x$. This calculation, although based on an incorrect independence assumption is often useful to get an estimate of the number of noncolliding paths that we can expect to find.

We observe that in [19] vertex-disjoint paths are found successively and the nodes that are used in paths for sourcedestination pairs $1, \ldots, i$ are eliminated entirely from the graph before finding the path for the $(i+1)$ st pair. The paper adroitly proves that at each stage the remaining graph has edges that are "approximately" i.i.d. (from the appropriate distribution).

$$
\begin{aligned}
& P(\text { path } 1 \text { collides with path } 2) \\
& \quad=P\left(\left(s_{1}=r_{2,1}\right) \cup \bigcup_{j=1}^{h-1}\left(r_{1, j}=r_{2, j-1} \cup r_{1, j}=r_{2, j} \cup r_{1, j}=r_{2, j+1}\right) \cup\left(d_{1}=r_{2, h-1}\right)\right) \\
& \quad \leq P\left(s_{1}=r_{2,1}\right)+\sum_{j=1}^{h-1} P\left(r_{1, j}=r_{2, j-1}\right)+\sum_{j=1}^{h-1} P\left(r_{1, j}=r_{2, j}\right)+\sum_{j=1}^{h-1} P\left(r_{1, j}=r_{2, j+1}\right)+P\left(d_{1}=r_{2, h-1}\right) \\
& \quad=\frac{3 h-1}{n-2} .
\end{aligned}
$$




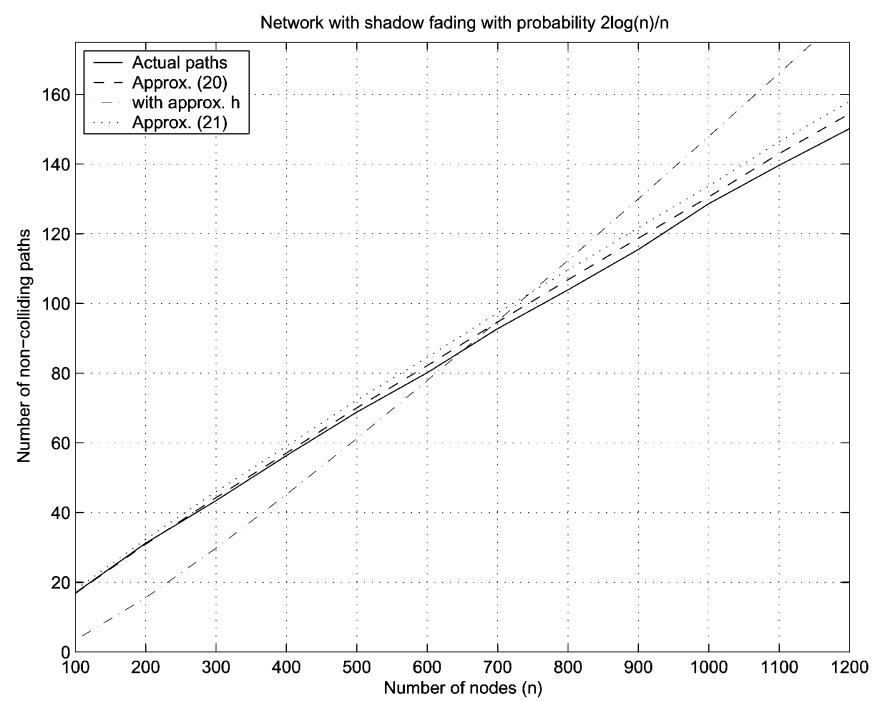

Fig. 4. Number of computer-found noncolliding paths versus $n$ for a shadowfading model with connection probability $2(\log n) / n$ (solid curve) versus $n$. Also shown are the approximation (22) (dashed curve closest to solid curve) and the approximation (23) (next-closest dashed curve) using values of $h$ obtained in the computer simulation. The dash-dotted curve is (22) computed using $h=$ $\log (n) / \log (n p)$.

The approximation we use previously deals with the loss of the i.i.d. property by simply ignoring it. Fig. 4 shows that the approximations (22) and (23) can be very accurate. The figure shows the number of computer-found noncolliding paths obtained in the shadow-fading model in Section VIII-A with link probability $p=2(\log n) / n$. (We provide more details about this simulation in Section X-B.) The most accurate approximation is obtained when the number of hops $h$ in (22) and (23) is also taken from the simulation. However, we may always approximate the number of hops before the simulation as $h=\log (n) / \log (n p)$. This final approximation is presented as the dashed-dotted curve.

\section{B. Simulations}

We revisit some of the examples analyzed in Section VIII to see how well our analytical predictions match computer-generated simulations. We begin with the shadow-fading network analyzed in Section VIII-A.

Fig. 5 shows the aggregate throughput and minimum SINR of a shadow-fading network as a function of the number of nodes $n$ in a computer-generated simulation where the channel connections are chosen as in Section VIII-A. The analytical results suggest that, for best throughput, we should choose $p=$ $\left(\log n+\omega_{n}\right) / n$ for $\omega_{n}$ going to infinity arbitrarily slowly. We therefore choose $p=2(\log n) / n$. The computer simulation begins by establishing a network of $n$ connections whose channels are drawn i.i.d. according to (15). Noncolliding paths (using the method described in Section X-A) are established and the minimum SINR obtained along the $i$ th path, denoted $\rho_{0, i}$, is found. We are assured that an SINR of $\rho_{0, i}$ can be supported by the path and we use this rather than the threshold of $\rho_{0}$ that has been used in the analysis. Although the threshold of $\rho_{0}$ is significant in obtaining the analytical throughput guarantee, we believe that the notion of the minimum SINR along a path is more useful in a

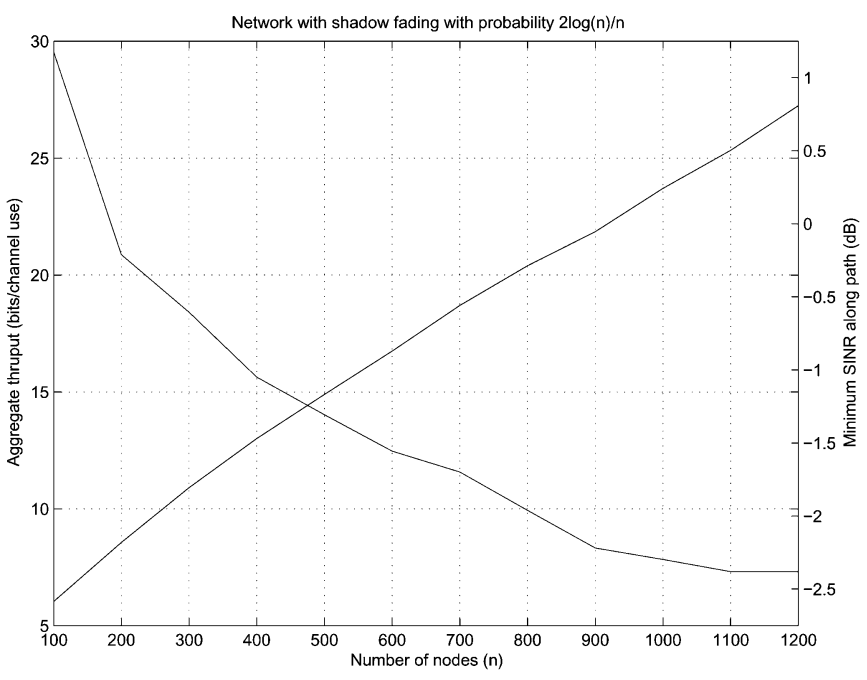

Fig. 5. Aggregate throughput and minimum SINR versus number of nodes $n$ in a shadow fading network with connection probability $p=2(\log n) / n$. The left $y$-axis contains the scale for this increasing function of $n$. We see that the aggregate throughput increases nearly linearly. The average SINR obtained along the paths (see scale on the right $y$-axis) drops with $n$, and according to the results in Section VIII-A should go to zero as $1 / \log \log n$.

practical system. Therefore, the quantity $\log \left(1+\rho_{0, i}\right)$ is then computed, weighted by the number of hops on path $i$, summed over $i$, and then normalized by the total number of hops contained in all paths. This gives a measure of the throughput per path, where paths that are longer (have more hops) count more heavily in the average. This throughput-per-path is then multiplied by the number of noncolliding paths and divided by the average number of hops to provide the aggregate throughput. Typically, we expect and observe only a small variation in the path lengths. Therefore, whether we divide by the average or largest path length does not make much difference.

The throughput shown in Fig. 5 is an increasing function of $n$ whose $y$-axis is labeled on the left. The minimum SINRs obtained along the $i$ th path $\rho_{0, i}$ are averaged over $i$ and displayed as a decreasing curve whose $y$-axis is labeled on the right. As predicted in Section VIII-A, the aggregate throughput grows nearly linearly. We see that the average SINR per path, decreases slowly with $n$, especially when $n$ is large; Section VIII-A shows that the SINR should go to zero as $1 / \log \log n$.

The following applies to all simulations described in this section: i) Computer simulations were repeated and averaged approximately 100-200 times, depending on the size of the network and variability of the results. ii) The nodes have unit transmit power $P=1$ and noise variance $\sigma^{2}=0.1$. Hence, on a unit channel and in the absence of interference, the SNR is $10 \mathrm{~dB}$. iii) We do not prescribe an SINR threshold. Rather, we accept any noncolliding path and use its resulting SINR in our averages. We believe this to be reasonable in practice (the threshold $\rho_{0}$ is only the guaranteed minimum). iv) The figures often show two plots; the aggregate throughput generally given by an increasing function of $n$ and whose scale is on the left $\mathrm{y} y$-axis, and the average minimum SINR generally given by a decreasing function of $n$ and whose scale is on the right $y$-axis. 


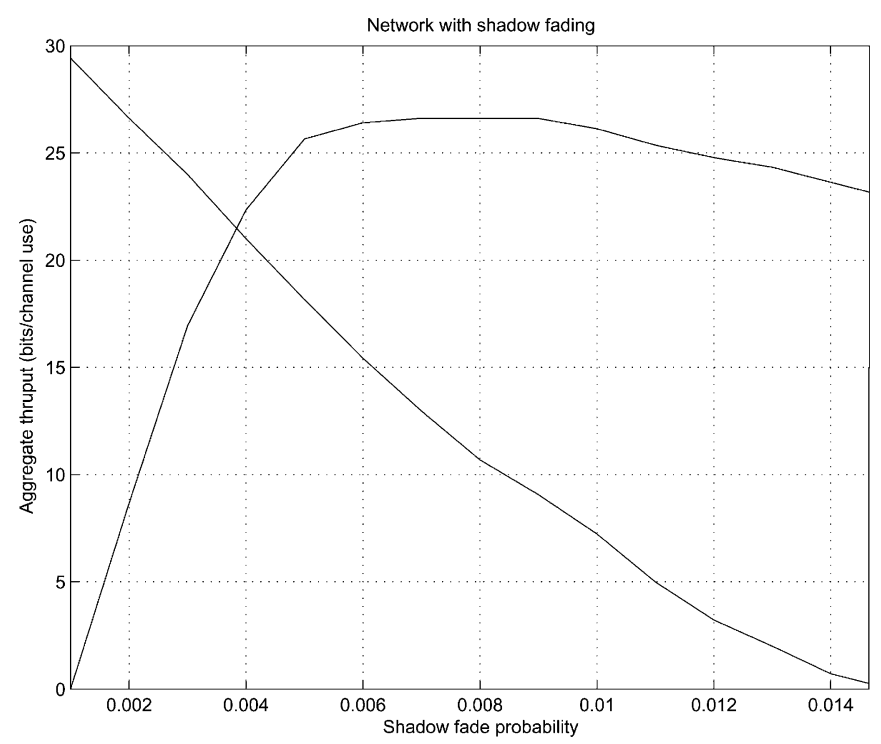

Fig. 6. Aggregate throughput and minimum SINR versus connection probability $p$ in a shadow-fading network of 1000 nodes. We see that the throughput is maximized at $p \approx 0.008$, which is not far from $(\log 1000) / 1000 \approx 0.0069$, the large- $n$ maximizing $p$ predicted in Section VIII-A.

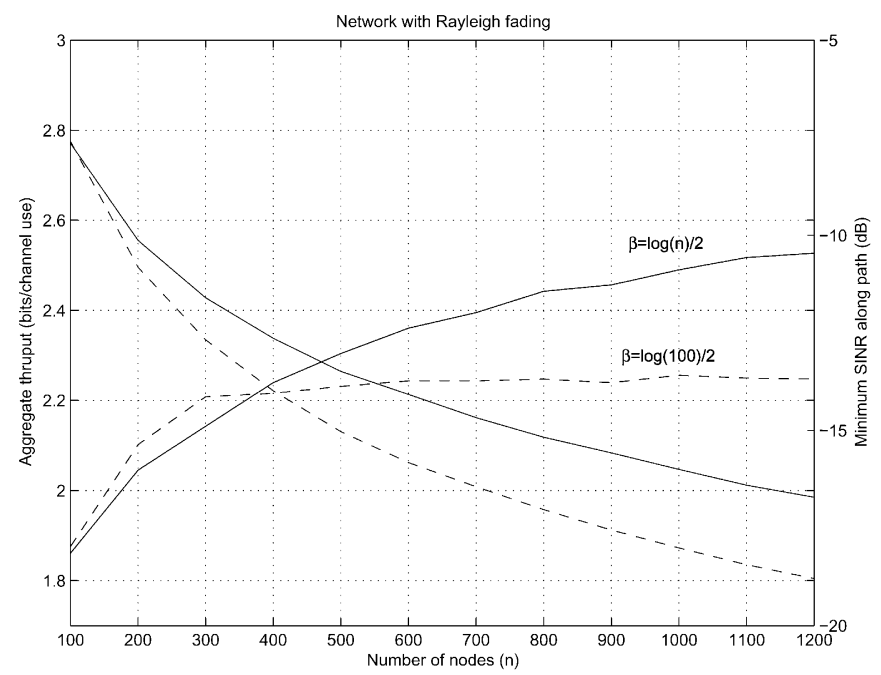

Fig. 7. Aggregate throughput and minimum SINR versus number of nodes $n$ in a network with exponential fading. We see that the throughput grows logarithmically using the optimum $\beta$ computed in Section VIII-D. The average SINR obtained along the paths decays approximately as $(\log n) / n$. Shown in dashed lines is the detrimental effect of choosing a constant $\beta=(\log 100) / 2$.

v) Although the analysis in the paper uses logarithms with base $e$, the throughputs in the figures are given in bits/channel-use.

Fig. 6 shows the aggregate throughput and minimum SINR of the same shadow-fading network, this time as a function of $p$ for a fixed $n=1000$ nodes. We see from the figure that the maximum throughput is attained when $p \approx 0.008$. Section VIII-A predicts that the maximum throughput is achieved when $p=$ $\left(\log n+\omega_{n}\right) / n=0.0069+\omega_{n} / n$. Ignoring the $\omega_{n}$ term, we see a good match between the theory and the simulation.

Fig. 7 shows the aggregate throughput and minimum SINR of a network with exponential fading analyzed in Section VIII-D as a function of $n$. For large enough $n$, the optimum threshold is $\beta=(\log n) / 2$ and $k$ should be chosen as large as possible.

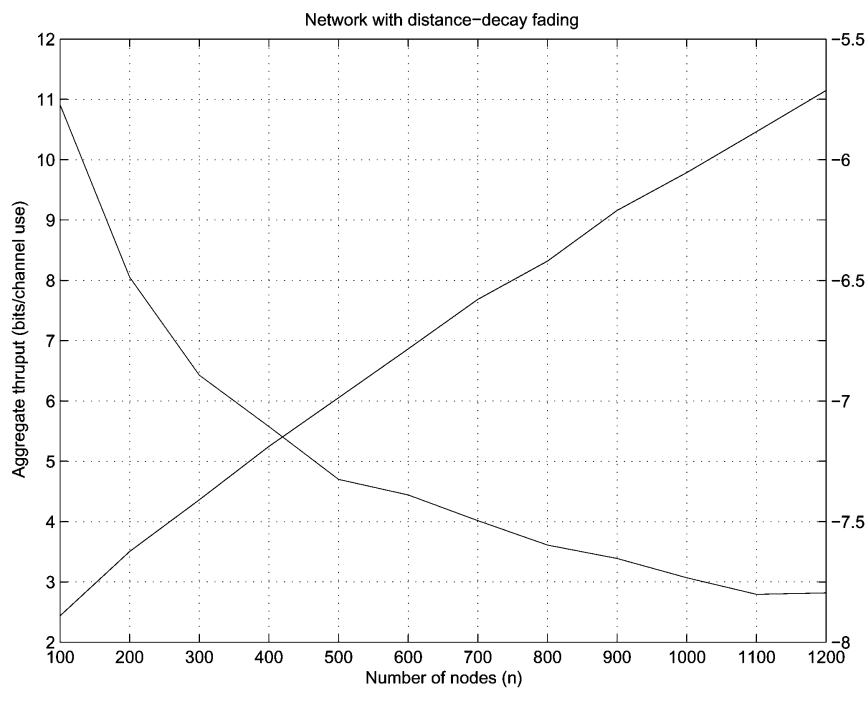

Fig. 8. Aggregate throughput and minimum SINR versus number of nodes $n$ in the decay-density network analyzed in Section VIII-B. Equation (20) (for $m>2$ ) predicts that the throughput should grow approximately linearly.

For purposes of illustration, we choose $k$ as large as possible, even for the relatively small values of $n$ that we consider. (In this particular example smaller values of $k$ can yield higher total throughput when $n$ is small.) The result is a throughput that grows approximately logarithmically with $n$, as predicted theoretically. The figure also shows that choosing a $\beta$ that is constant has a detrimental effect on the throughput. Similarly, choosing a $\beta$ that grows faster than logarithmically would also be detrimental.

Fig. 8 shows the aggregate throughput and minimum SINR of the decay-density network (as a function of $n$ ) described in Section VIII-B. The parameters used in the simulation are $d=$ $1, \Delta=1$, and $m=3$. This is equivalent to placing nodes with unit spacing in a two-dimensional lattice and assuming a power-decay that decreases as $1 / r^{3}$. The figure shows that the throughput grows approximately linearly, as predicted by (20).

These simulations show that Theorem 1, although designed for large $n$, is also an accurate predictor for finite $n$.

\section{Conclusion}

Our model for shared-medium wireless networks uses channels chosen according to a common distribution. We have devised a method of operating this network using relays and provided an achievable aggregate throughput as a function of the distribution. Distributions that have a certain sparsity of "good" connections seem to fare best and provide near-linear throughputs. We show that there exists an optimum amount of shadow fading that a network should have-any more or any less degrades the throughput. We hope that these results provide guidelines to the design of networks including, paradoxically, possible obstacle placement if the network is "over-connected."

We have given a brief description of an upper bound on the achievable throughput. We do not generally know how sensitive our throughput results are to relaxing the i.i.d. assumption on the channel coefficients. A case where the channel coefficients are independent but have distribution that depends on distance was examined in Section VIII, where we argued that at the low 
connection probabilities that we require, the sensitivity to distance was low. It remains to be seen whether this sensitivity is low more generally.

More practical issues remain to be addressed. For instance, the scheduling algorithm used in the simulations requires centralized knowledge of the channel connections. In a practical network we would expect sources and destinations to have knowledge of their own connections and determine suitable relaying paths. Ideally, we would then like the network to generate a noncolliding schedule in a decentralized manner. Another issue of interest is that of modeling a network that has both randomness and geometric distance decay laws. One such model is proposed in [28].

\section{REFERENCES}

[1] F. Baccelli, M. Klein, M. Lebourges, and S. Zuyev, "Stochastic geometry and architecture of communication networks," J. Telecommun. Syst., vol. 7, pp. 209-227, 1997.

[2] B. Bollobás, Random Graphs, 2nd ed. Cambridge, U.K.: Cambridge Univ. Press, 2001.

[3] O. Dousse and P. Thiran, "Connectivity versus capacity in dense ad hoc networks," in Proc. 23rd INFOCOM, Hong Kong, Mar. 2004, pp. 476-486.

[4] G. J. Foschini, "Layered space-time architecture for wireless communication in a fading environment when using multi-element antennas," Bell Labs. Tech. J., vol. 1, no. 2, pp. 41-59, 1996.

[5] M. Gastpar and M. Vetterli, "On the capacity of wireless networks: The relay case," in Proc. 21st INFOCOM, New York, Jun. 2002, pp. $1577-1586$.

[6] M. Grossglauser and D. Tse, "Mobility increases the capacity of ad-hoc wireless networks," IEEE/ACM Trans. Netw., vol. 10, no. 4, pp. 477-486, Aug. 2002.

[7] R. Hekmat and P. van Mieghem, "Degree distribution and hopcount in wireless ad-hoc networks," in Proc. 11th IEEE Int. Conf. on Networks (ICON), Sydney, Australia, Sep. 2003, pp. 603-609.

[8] R. Hekmat and P. V. Mieghem, "Study of connectivity in wireless ad-hoc networks with an improved radio model," in Proc. 2nd Workshop on Model. and Optim. in Mobile, Ad Hoc and Wireless Networks, Cambridge, U.K., Mar. 2004, pp. 142-151.

[9] O. Léveque and E. Telatar, "Information theoretic upper bounds on the capacity of large extended ad-hoc wireless networks," IEEE Trans. Inf. Theory, vol. 51, no. 3, pp. 858-865, Mar. 2005.

[10] M. Franceschetti, O. Dousse, D. N. C. Tse, and P. Thiran, "On the throughput capacity of random wireless networks," IEEE Trans. Inf. Theory, 2004, submitted for publication.

[11] P. Gupta and P. R. Kumar, "The capacity of wireless networks," IEEE Trans. Inf. Theory, vol. 46, no. 3, pp. 388-404, Mar. 2000.
[12] _ - "Toward an information theory of large networks: An achievable rate region," IEEE Trans. Inf. Theory, vol. 49, no. 8, pp. 1877-1894, Aug. 2003

[13] I. E. Telatar, "Capacity of multi-antenna Gaussian channels," Eur. Trans. Telecom., vol. 10, pp. 585-595, Nov. 1999.

[14] O. Tonguz and G. Ferrari, "Connectivity and transport capacity in ad hoc wireless networks," in Proc. 32nd IEEE Communication Theory Workshop, Mesa, AZ, Apr. 2003.

[15] L.-L. Xie and P. R. Kumar, "A network information theory for wireless communication: Scaling laws and optimal operation," IEEE Trans. Inf. Theory, vol. 50, no. 5, pp. 748-767, May 2004.

[16] S. Toumpis and A. Goldsmith, "Capacity bounds for large wireless networks under fading and node mobility," in Proc. 41st Allerton Conf. Communication, Control and Computing, Monticello, IL, Oct. 2003, pp. 1369-1378.

[17] S. Weber, X. Yang, J. Andrews, and G. de Veciana, "Transmission capacity of wireless ad hoc networks with outage constraints," IEEE Trans. Inf. Theory, vol. 51, no. 12, pp. 4091-4102, Dec. 2005.

[18] F. Chung and L. Lu, "The diameter of random sparse graphs," $A d v$. Appl. Math., vol. 26, pp. 257-279, 2001.

[19] A. Z. Broder, A. M. Frieze, S. Suen, and E. Upfal, "An efficient algorithm for the vertex-disjoint paths problem in random graphs," in Proc 7th Symp. Discrete Algorithms, Atlanta, GA, 1996, pp. 261-268.

[20] T. H. Cormen, C. E. Leiserson, R. L. Rivest, and C. Stein, Introduction to Algorithms, 2nd ed. Cambridge, MA: MIT Press, 2001.

[21] J. M. Abram and I. B. Rhodes, "Some shortest path algorithms with decentralized information and communication requirements," IEEE Trans. Autom. Control, vol. AC-27, no. 3, pp. 570-582, Jun. 1982

[22] F. Xue, L.-L. Xie, and P. R. Kumar, "The transport capacity of wireless networks over fading channels," IEEE Trans. Inf. Theory, vol. 51, no. 3, pp. 834-847, Mar. 2005.

[23] A. Jovicic, P. Viswanath, and S. R. Kulkarni, "Upper bounds to transport capacity of wireless networks," IEEE Trans. Inf. Theory, vol. 50, no. 11 , pp. 2555-2565, Nov. 2004.

[24] D. Miorandi and E. Altman, "Coverage and connectivity of ad hoc networks presence of channel randomness," in Proc. IEEE INFOCOM 2005, pp. 491-502.

[25] P. Balister, B. Bollobás, and M. Walters, "Continuum percolation with steps in an annulus," Ann. Appl. Probab., vol. 14, no. 4, pp. 1869-1879, 2004.

[26] M. Franceschetti, L. Booth, M. Cook, J. Bruck, and R. Meester, "Continuum percolation with unreliable and spread out connections," J. Statist. Phys., vol. 118, no. 3/4, Feb. 2005.

[27] R. Gowaikar, B. Hochwald, and B. Hassibi, "An achievability result for random networks," in Proc. IEEE ISIT 2005, Adelaide, Australia, pp. 946-950.

[28] R. Gowaikar and B. Hassibi, "On the achievable throughput in twoscale wireless networks," in Proc. IEEE Int. Symp. Information Theory, Seattle, WA, Jul. 2006, to be published.

[29] M. Penrose, Random Geometric Graphs. Oxford, U.K.: Oxford Univ. Press, 2003. 